\title{
Dynamic CFD Simulations of the MEADS II Ballistic Range Test Model
}

\author{
Eric C. Stern* \\ NASA Ames Research Center, Moffett Field, CA 94035 \\ Alan M. Schwing ${ }^{\dagger}$ \\ NASA Johnson Space Center, Houston, TX, 77584 \\ Joseph M. Brock ${ }^{\ddagger}$ \\ AMA Inc., Moffett Field, CA 94035 \\ Mark Schoenenberger $\S$ \\ NASA Langley Research Center, Hampton, VA 23681
}

\begin{abstract}
Dynamic, viscous, free-to-oscillate simulations of the Mars Entry Atmospheric Data System (MEADS) ballistic range model are performed using two different flow solvers, OVERFLOW and US3D. At the time of publication, data from the ballistic range experiment was not yet available, so the current work serves as a code-to-code exercise. Results from the analysis show good agreement between the predicted static aerodynamic coefficients for each solver. Both codes predict damped pitch oscillations for Mach 3.0 with initial amplitudes of $5^{\circ}$ and $30^{\circ}$, as well as for Mach 1.5 with initial amplitude of $30^{\circ}$. The two solvers predict undamped pitch oscillations for Mach 1.5 with initial amplitude of $5^{\circ}$. For most cases, US3D predicts less damping than OVERFLOW. The difference is attributed to higher pressures in the separated region of the wake, and the resultant effect on the backshell contribution to the pitching moment.
\end{abstract}

\section{Introduction}

The NASA Engineering and Safety Center (NESC) has commissioned a ballistic range test campaign to perform analysis in support of the Mars Entry Descent and Landing Instrumentation-2 (MEDLI2) experiment, which will fly on the Mars 2020 mission. For this flight experiment, a flush air data system, similar to the one used for the Mars Science Laboratory, will provide an estimate of vehicle attitude and velocity over the course of its trajectory. This system is the second Mars Entry Atmospheric Data System or MEADS-II. ${ }^{1,2}$ The current ballistic range campaign will provide unique data for comparison to CFD predictions in that the ballistic range models will be instrumented with pressure transducers, enabling greater resolution of the forebody and aftbody contributions to the total aerodynamic forces. Additionally, pressure transducers located on the model backshell will allow comparison of the predicted wake flow environment, which is believed to have a significant effect on the dynamic stability of blunt bodies in the low-supersonic to transonic regime, ${ }^{3-5}$ to that of the experiment.

The NESC has further identified a need for the ability to characterize the dynamic stability of entry vehicles using high-fidelity computational fluid dynamics (CFD) tools. Typical blunt body entry vehicles become dynamically unstable in the low supersonic regime (i.e. < Mach 2.5). Margins associated with extrapolating experimentally derived dynamic stability parameters to flight place constraints on the entry sequence by

\footnotetext{
${ }^{*}$ Research Engineer, Thermal Protection System Materials Branch, Member AIAA.

${ }^{\dagger}$ Engineer, Applied Aeroscience and CFD Branch, Member AIAA.

¥Associate Research Scientist, Aerothermodynamics Branch, Member AIAA

$\S$ Aerospace Engineer, Atmospheric Flight and Entry Systems Branch, Member AIAA
} 
requiring parachute deployment at higher Mach numbers, and/or active guidance and control. Furthermore, the physical mechanisms of dynamic instability for entry vehicles are not well understood. Having a reliable computational capability for simulating capsule dynamics would allow researchers to examine the flow physics in this regime and thereby gain insight into the design factors that influence the stability.

In the current work, we assess and compare the capabilities of two of NASA's flow solvers, US3D and OVERFLOW, applied to on-going ballistic range tests in support of MEADS II development. We will begin by providing a brief overview of the ballistic range experiment insofar as the details are relevant to the current investigation. We then provide an overview of each of the flow solvers, as well as their respective implementations of rigid body dynamics capabilities. The analysis will first look at comparisons of static aerodynamic coefficients before looking in greater detail at the predicted dynamic behavior.

\section{Ballistic Range Experiment}

The ballistic range models were shot through the Test and Evaluation Facility (TEF), operated by the US Army Research Laboratory, USARL, at Aberdeen Proving Ground. The TEF is an indoor ballistic range, instrumented with 25 orthogonal shadowgraph stations spanning approximately $200 \mathrm{~m}$ downrange. Infrared sensors detect the models as they fly down the range and initiate spark light sources which produce shadowgraphs images on reflective screens on a side-wall and ceiling of the range. Medium format cameras record the images to measure the position and orientation of the model versus time. Six-degree-of-freedom trajectory simulations are fit to these position/orientation data. This is typically done to identify aerodynamic coefficients of the model (dynamic and static). The aerodynamic coefficients of the Mars Science Laboratory entry vehicle have been measured in previous ballistic range tests. ${ }^{6}$ The shadowgraph data is used to provide trajectory data to correlate with the onboard pressure measurements.

Four instrumented ballistic range models were built for this test. The models were shot from a $120 \mathrm{~mm}$ power-charge gun at an initial velocity of approximately $1000 \mathrm{~m} / \mathrm{s}$. The models were held in four-petal sabots in the gun. The sabots held the model at the desired initial angle during launch. Upon exit from the gun, the petals fall away and the model proceeds into the range. The muzzle velocity was chosen to have the models reach the first data station at a Mach number near 2.5. The models then decelerate down the range and are caught just after shadowgraph station 15 (of 25), approximately $112 \mathrm{~m}$ downrange from station 1 . The models impact and are brought to rest within a stack of boxes filled with a material similar to particle-board and bails of cardboard. The models must be recovered so that data can be downloaded off the non-volatile memory onboard. The models were launched at initial total angles of attack of 0 degrees and 10 degrees.

The models each had one 0-500psi forebody pressure port located at the model nose. They each had two or three 0-25psi transducers on the backshell, located approximately midway down the first and second cones aft of the max diameter. The azimuthal locations of the ports varied; the different port arrangements were selected to look for temporal and spatial variations of the wake pressures during flight. A total of seven shots were made, with some models being fired twice or three times, though not all transducers were functioning for repeat shots. Useful data was recovered from three separate ballistic range shots, and work continues to recover data from two of the models.

Preliminary mass properties are included in Tab. 1. The center of gravity for the model is on the centerline of the model, $27 \mathrm{~mm}$ from the forward-most point of the heatshield $\left(x_{c g} / D=0.3\right)$.

\begin{tabular}{cc}
\hline Moments of Inertia & {$\left[\mathrm{g} \times \mathrm{mm}^{2}\right]$} \\
\hline $\mathrm{I}_{x}$ & 916,000 \\
$\mathrm{I}_{y}$ & 638,000 \\
$\mathrm{I}_{z}$ & 638,000 \\
\hline Mass & $1370[\mathrm{~g}]$ \\
\hline
\end{tabular}

Table 1. Notional mass properties for MSL ballistic range models.

The coordinate system that will be used to compute aerodynamic coefficients is illustrated in Fig. 1(b). Note that all simulations performed for the current work are constrained to the pitch plane, and therefore the only angle used in this figure is $\alpha$. Figure 1(a) shows the outer mold line (OML) for the test article simulated 
in the current work. Note that the dimension and mass properties for the simulation are "representative" of the models used for the experiment and are not exact.

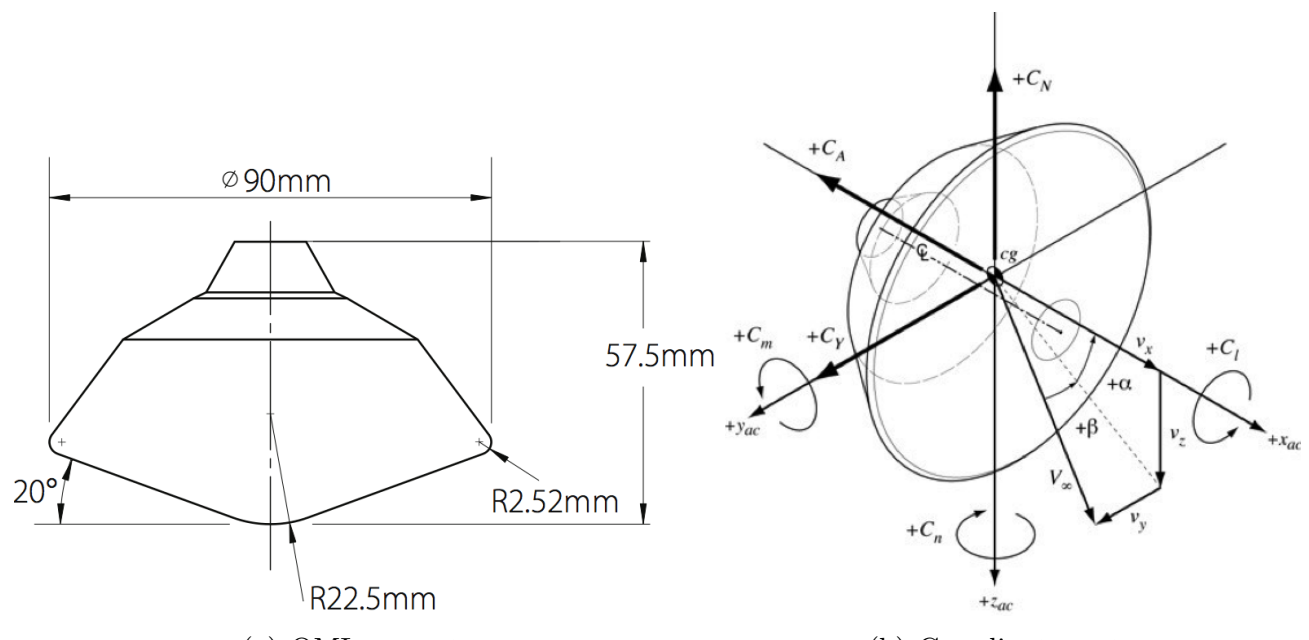

(a) OML geometry

(b) Coordinate system

Figure 1. Simplified model geometry and coordinate system for ballistic range test.

\section{Flow Solvers}

In this section, we briefly describe the two flow solvers, US3D and OVERFLOW, used in this study. The implementation of rigid body dynamics solvers within each code is discussed in a subsequent section. For the current work, we have not made any effort to run the two solvers with equivalent numerics or modeling settings. The two codes use fundamentally different spatial discretizations so a true one-to-one comparison is not strictly possible. Therefore, in general, we have opted for respective settings which favor computational robustness and efficiency, as well as adherence to standard practices for each code for this application. Future work will entail more detailed examination of the effect of numerical and modeling choices on the predicted dynamics.

\section{A. US3D}

US3D is an unstructured, three-dimensional, finite-volume, parallel, implicit Navier-Stokes solver developed at the University of Minnesota. ${ }^{7,8}$ It can be considered the unstructured descendent of the Data Parallel Line Relaxation ${ }^{9}$ (DPLR) code, in wide use at NASA, as well as across academia and industry. Originally developed for simulating hypersonic flow with thermochemical non-equilibrium, it has recently - thanks to the implementation of low-dissipation numerical fluxes ${ }^{10}$ - been applied increasingly to the high-resolution simulation of massively separated flows. ${ }^{11}$ As is discussed in a later section of the current work, the fluid dynamics of the wake has a significant effect on the dynamic stability of the vehicle.

\section{Grid Generation}

One of the critical differences between US3D and OVERFLOW is that US3D exclusively uses body-fitted meshes. In practice this means that much of the user's time is spent generating a high-quality mesh to provide adequate resolution of important flow features. This is in contrast to OVERFLOW which uses overset grid techniques thereby allowing a good deal more automation of the grid generation process.

For the simulations in the current work, the same grid was used for all cases. The grid is generated using the commercial package GridPro. ${ }^{12}$ Some visualizations of the US3D mesh can be seen in Fig. 2. Because we use the same grid for a range of Mach numbers, as well as pitch angles, we have added refinement to accommodate, both ahead of the body, and aft. Additionally, for performing dynamic simulations, it is desirable to have greater distance between the body and domain boundaries than would ordinarily be 
required. This is to accommodate the grid deformation that is used in US3D to perform rigid body dynamic simulations. The grid used for these simulations had a total of 20 million cells. Typical grid spacings in the wake are approximately $2 \mathrm{~mm}$. The first cell wall spacing was set to be $1 \mu \mathrm{m}$, which results in a $y^{+}<1$ for the entire vehicle surface.

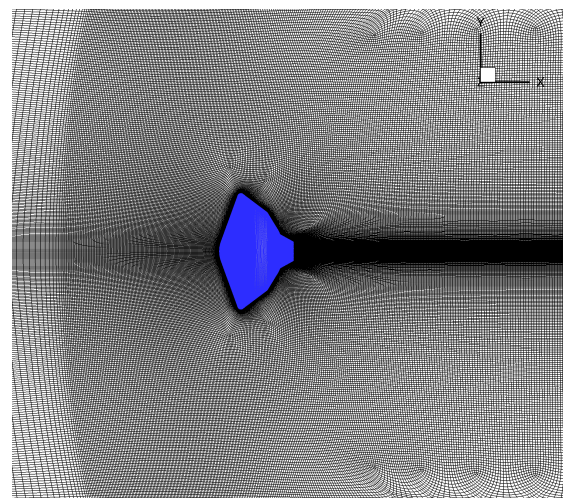

(a) Full

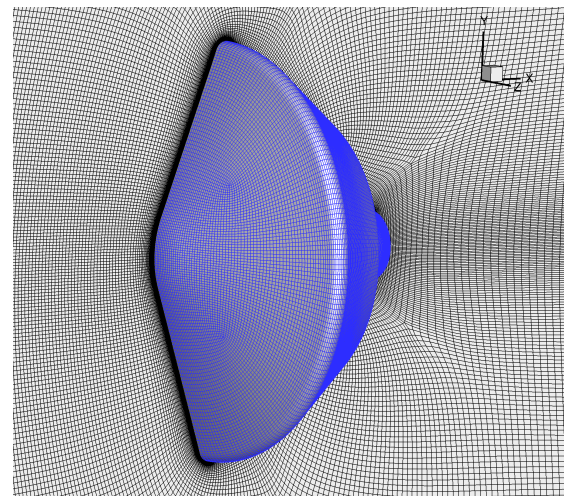

(c) Forebody

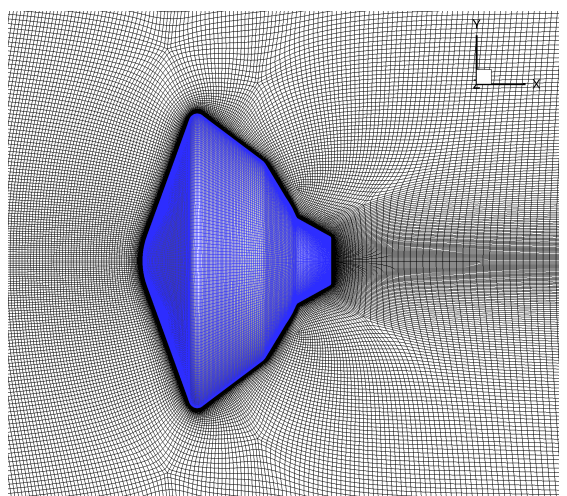

(b) Close-up side

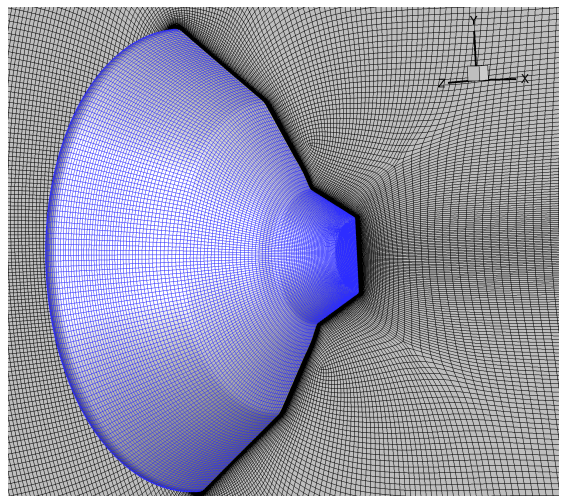

(d) Backshell

Figure 2. Grid used for US3D simulations.

\section{Numerical Methods}

In this study, the modified Steger-Warming ${ }^{13}$ flux-vector splitting scheme is used. A Monotone Upwind Scheme for Conservation Laws ${ }^{14}$ (MUSCL) extrapolation is used to achieve second order accuracy in regions without a discontinuity. Time integration is performed using the Full Matrix Data-Parallel Method ${ }^{15}$ point implicit method. The global CFL number for this scheme is set such that a local CFL number in the unsteady wake of approximately unity was achieved, which results in an average timestep of approximately $1.25 \mu \mathrm{s}$. Additionally, the flow in all cases is assumed to be fully turbulent, with the turbulent transport coefficient modeled using Detached Eddy Simulation (DES), ${ }^{16}$ with the Spalart-Allmaras ${ }^{17}$ one-equation model with the Catris-Aupoix ${ }^{18}$ compressibility correction used to account for turbulent diffusion at sub-grid scales.

\section{Timing Metrics}

All dynamic simulations in the current work were run on 256 AMD Opteron processors (16 cores per CPU, 4 CPU's per compute node). Average timing metrics were consistent across all cases. For dynamics case 1 (see below), the average computational cost per iteration was $0.194 \mathrm{CPU}$ hours. This resulted in an average computational cost per flow time $\left(t_{\text {flow }}=D / V_{\infty}\right)$ of $13.71 \mathrm{CPU}$ hours. Note that, because the flow time is doubled for the lower Mach cases (Cases 3 and 4), and the timestep was held fixed, the cost per flow time for these cases was doubled accordingly. To give an idea of the cost of performing the dynamic analysis 
described in subsequent sections, the associated cost with simulating a full pitch oscillation cycle at Mach 3.0 is $\approx 4250 \mathrm{CPU}$ hours.

\section{B. OVERFLOW}

OVERFLOW is a three-dimensional, finite-difference, parallel, implicit Navier-Stokes' solver capable of using overset grid topologies. It was originally developed at NASA Ames Research Center, and is currently developed and maintained by NASA Langely Research Center. ${ }^{19}$ For moving-body simulations, it uses the Geometry Manipulation Protocol (GMP) tool to modify the relative positions of the computational grids and allow for constrained or unconstrained motion. ${ }^{20}$

\section{Grid Generation}

Structured, overset grids of the capsule and the surrounding domain were generated with the Chimera Grid Tools (CGT). ${ }^{21,22}$ CGT allows for the creation of grid scripts for parametrically controlled grids for complex shapes. The script system holds fixed the surface and volume spacing, ensures bounded and consistent grid stretching ratios, and allows for rapid re-meshing with updated inputs.

OVERFLOW's built-in Domain Connectivity Function (DCF) was utilized to perform hole cutting and calculation of interpolation stencils between overset grids. ${ }^{23}$ regions of the overlapping grids are blanked due to intersection with solid bodies (the MSL capsule) and also in regions of transition between body-fitted and off-body grids. Two types of off-body grids are used for this analysis: manual and automatically generated box grids. The manual box grids include a shock box to capture the shock and near-body flow and a wake box grid designed to contain the capsule's subsonic wake. The automatic box grids encapsulate the body-fitted and manual box grids, which extend into the far field as shown in Fig. 3.

Figure 3 shows several views of the OVERFLOW grid system. The automatic off-body grids are shown in blue, the wake box grid is in green, the shock box grid is in gray, and the body-fitted grids are black. In Fig. 3(a), the entire grid system is shown. The automatic off-body grids are of varying grid density to efficiently grow from the fine spacings necessary near the body to larger spacings at the boundary. Figure 3(b) presents a closer view of the manual box grids and the imbedded capsule geometry and grid.

By using the DCF capabilities of OVERFLOW, the component grids in the system can be arbitrarilly translated and rotated prior to hole cutting. This is useful for creating grids with the capsule at different angles of attack and is required in order to model motion during a simulation. Figures 3(c) and (d) show a close-up of the shock box and capsule grids at two angles of attack. Motion of the capsule is modeled by motion of the body-fitted grids inside of the manual box grids, which remain oriented with the freestream flow.

With respect to grid spacings, the wake box uses a constant spacing at its core of $0.017 D_{\circ}$ (normalized to the MSL diameter). The shock box is finer with its core of cartesian cells having an isotropic spacing one third of what is in the wake box. The entire grid system has a total of 36 million points in the manual boxes and body-fitted grids and an additional 200,000 in the automatic off-body box grids.

For the static results, grid adaptation was used to increase the refinement in bow shock, however it only influenced the integrated aerodynamics coefficients by less than half of a percent. Further refinement did not reduce the error commensurate with the cost. Test cases with refinement for dynamic simulations showed that it did not have a significant effect on results and was not used. Figure 4 shows an illustration of the additional refinement added by the solver for two static Mach 3.0 simulations. Refinement was constrained to only the forward portion of the shock box grid.

\section{Numerical Methods}

For this work, the solver was run in a time-accurate mode with 5 Newton sub-iterations per time step. The Harten-Lax-van Leer-Einfeldt (HLLE ++) upwind scheme was used for discretization of the advective terms. Implicit time advancement used the symmetric successive over-relaxation (SSOR) algorithm. ${ }^{24}$ This work employs a hybrid DES model based on the two-equation Shear Stress Transport turbulence model by Menter. ${ }^{16,25}$ The flowfield was assumed to be fully turbulent. 


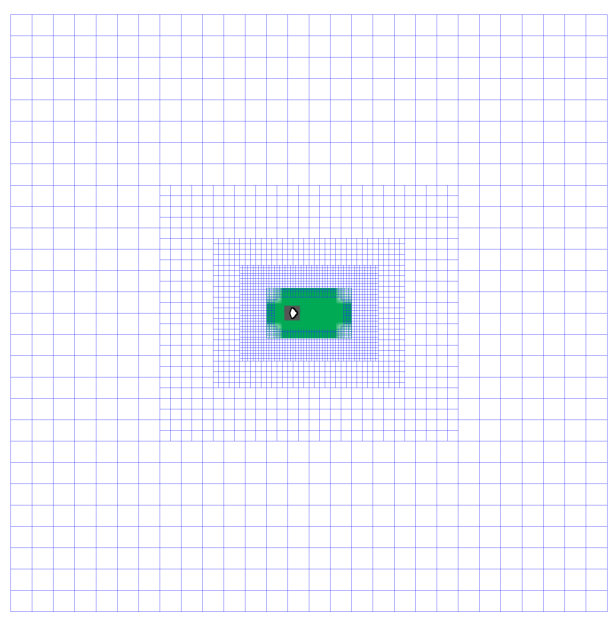

(a) Entire grid system

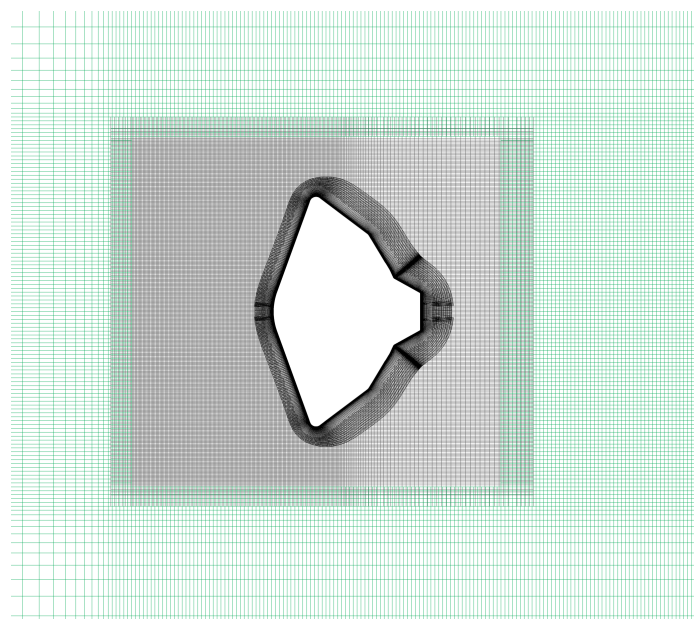

(c) Shock box and capsule $\left(\alpha=0^{\circ}\right)$

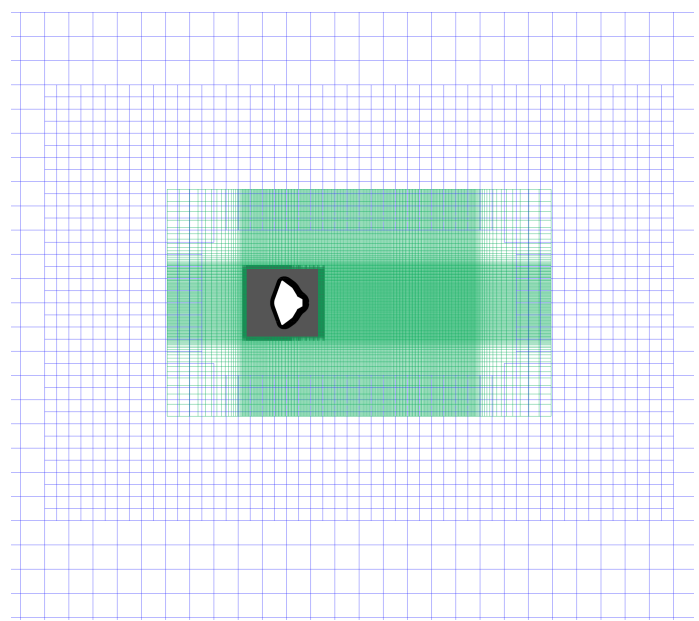

(b) Manual boxes and capsule

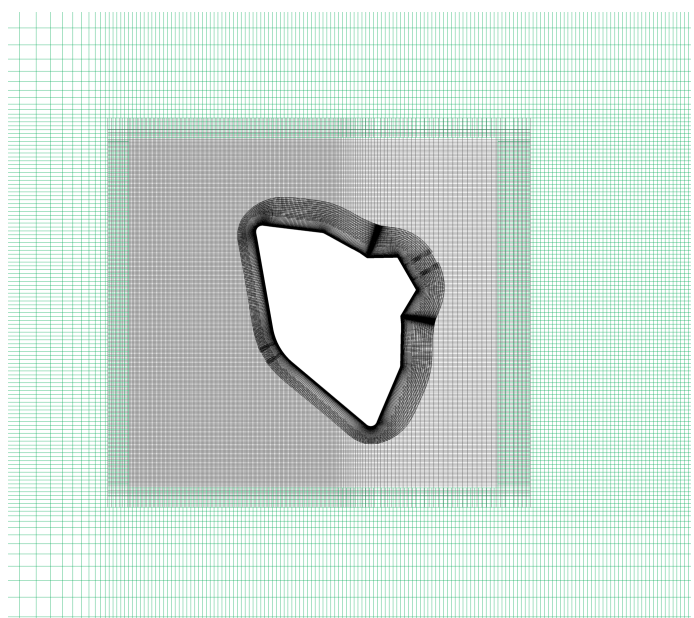

(d) Shock box and capsule $\left(\alpha=30^{\circ}\right)$

Figure 3. Illustration of computational grid topologies for OVERFLOW solutions.

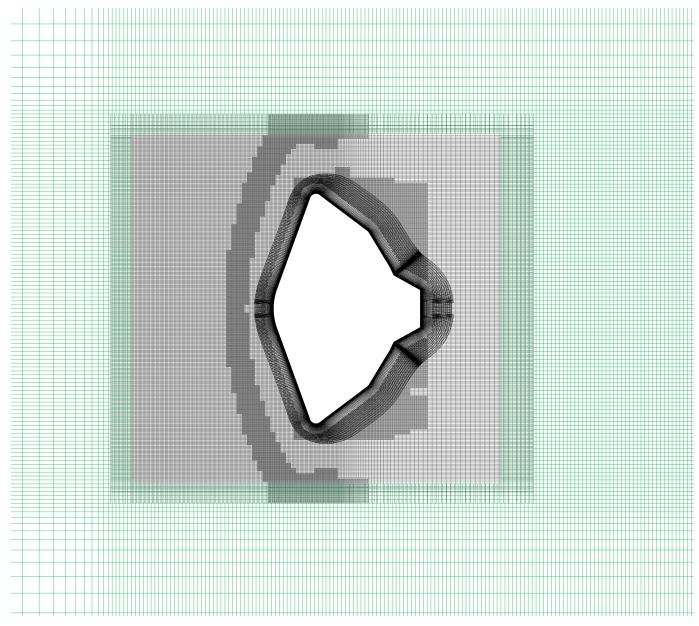

(a) Shock box and capsule $\left(\alpha=0^{\circ}\right)$

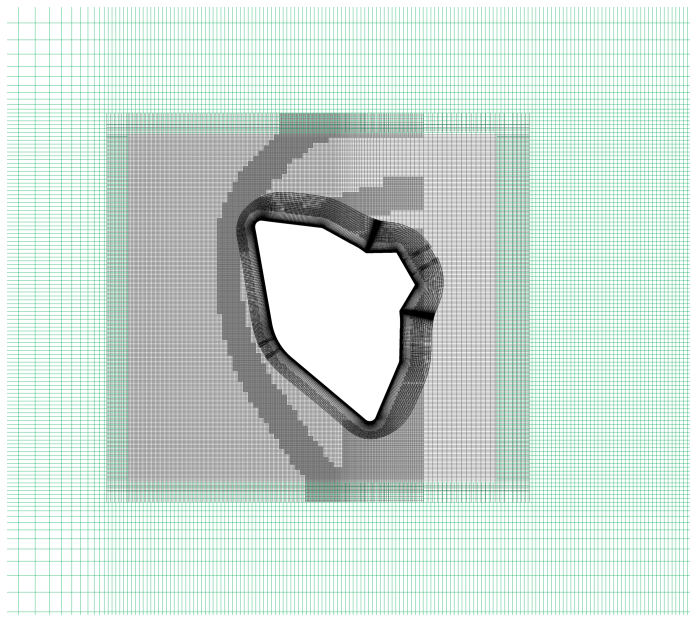

(b) Shock box and capsule $\left(\alpha=30^{\circ}\right)$

Figure 4. Illustration of computational grid topologies for OVERFLOW solutions using adaptation. 
Three levels of full-multigrid were used for flowfield initialization. Once a steady-state solution was reached, the simulation was run in a time-accurate mode to resolve unsteadiness.

Similar to the US3D solutions, dynamic simulations begin with a statistically converged static simulation. We found that a normalized time step of $0.01\left(\frac{\Delta t V_{\infty}}{D}\right)$ was small enough to achieve temporally converged results for moving-body simulations with respect to integrated aerodynamics. This timestep was used for both the static and moving-body results shown below.

\section{Timing Metrics}

The Case 1 simulation was run on an SGI ICE system using 192 Intel Haswell processors (12-core at 2.30GHz) linked via FDR infiniband. Over the entire simulation, the average processing time was 0.44 CPU hours per iteration. This translates into an average of $44 \mathrm{CPU}$ hours per flow time since the timestep was fixed at $0.01 \frac{\Delta t V_{\infty}}{D}$. Due to the timestep specification, the timings are identical for all other cases run on a similar architecture.

\section{Dynamic Simulation Methodology}

In this section, we briefly describe the approach for performing dynamic simulation utilized by each code.

\section{A. Moving Mesh Approach using US3D}

The US3D flow solver uses body-fitted unstructured grids. The approach taken for moving the mesh is to define a rigid sphere near the vehicle and encompassing the vehicle itself. Beyond this spherical region, there is a region of the grid that will be allowed to deform as the vehicle rotates. Finally, there is another rigid region between the flexible region and the outer boundary of the mesh. This approach is illustrated in Fig. 5 for the ballistic range model. Typically, the nominal mesh will be generated for the trim angle of attack, as seen in the left side of the figure.

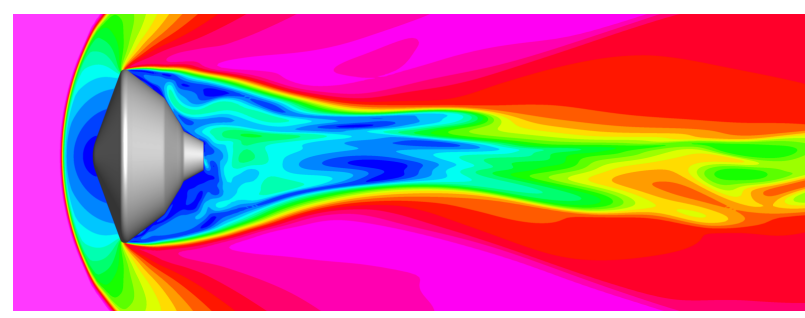

(a) Mach contours, $\alpha=0^{\circ}$

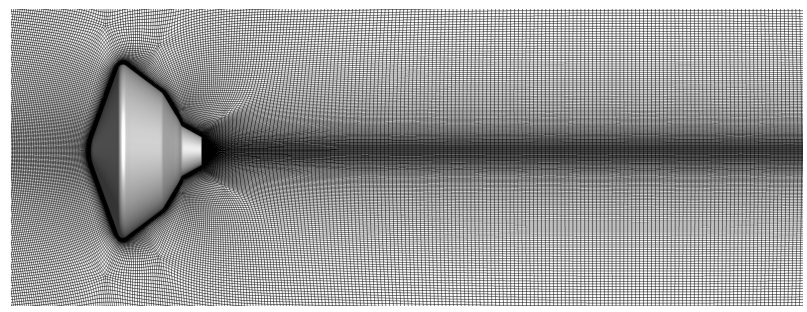

(c) Nominal mesh, $\alpha=0^{\circ}$

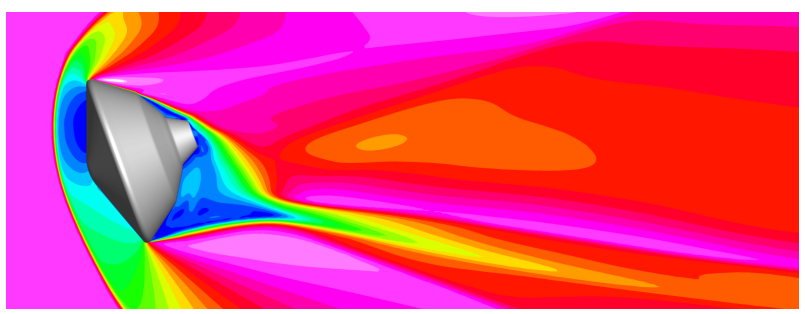

(b) Mach contours, $\alpha=20^{\circ}$

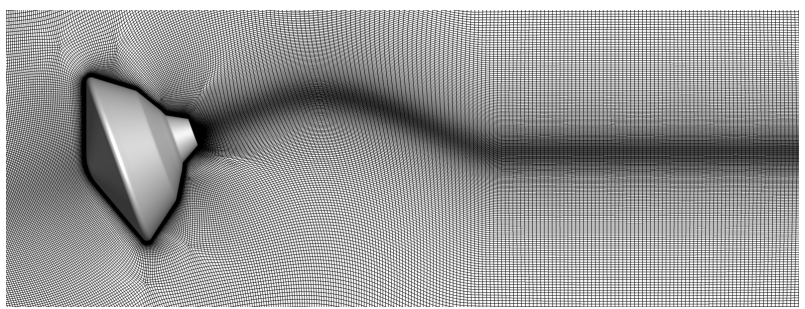

(d) Deformed mesh, $\alpha=20^{\circ}$

Figure 5. Illustration of the moving mesh approach in the US3D flow solver. The frames on the left show the nominal undeformed grid at the trim angle of attack. The frames on the right show the mesh after rotating the vehicle by $20^{\circ}$ in the pitch plane.

For a given timestep, the angular and translational accelerations for the vehicle are determined by the aerodynamic loads. The displacements of the nodes within the mesh are determined by the rotation of the vehicle, as well as the distance from the node to the vehicle center-of-gravity (CG). We wish for the nodes in the near-body region to rotate with the body, while the nodes in the region extending the domain boundary remain static. The nodes in the interstitial deformable region simply undergo a weighted rotation based 
on their position between the inner and outer shells. A limitation of this approach is that the it cannot accommodate unlimited deflections. As the grid is moved further from trim, grid cells within the flexible layer will become more and more skewed which adversely affects the quality of the solution. Since entry vehicles are designed such that there should not be large excursions from trim during the decent phase, this may not be a concern. Translational degrees of freedom are handled using a reference frame velocity which modifies the fluxes in the finite volume scheme. A more detailed description of the mesh motion technique can be found in Stern et al., ${ }^{26}$ and its accompanying references.

\section{B. Moving Mesh Approach using OVERFLOW}

In OVERFLOW, the mechanics of the moving mesh allow for the capsule grids to rotate and translate independent of the background box grids. This provides flexibility in vehicle attitude without affecting the size or quality of the grid cells. Every timestep the solver recalculates overset boundaries and interpolation stencils based on the updated positions of all relevant components. Prior to this update, the integrated forces and moments on the vehicle dictate to the solver the accelerations to place on the capsule grids so as to update its position.

Figure 6 shows an example of constrained pitch motion from a dynamic OVERFLOW solution. The initial position of the capsule was rotated to $30^{\circ}$ and allowed to freely pitch in a Mach 3.0 flow. Shown in the image are the resulting grids and Mach contours of the flow at two instances of time, the first when the capsule passes through $0^{\circ}$, and the second when the capsule passes through $20^{\circ}$ angles of attack. The extent of the grid motion is limited to the attitude of the capsule with the surrounding box grids remaining fixed in space. For a solution involving translation of the capsule, the two manual box grids translate with the body and the automatic box grids are adapted to track the movement.

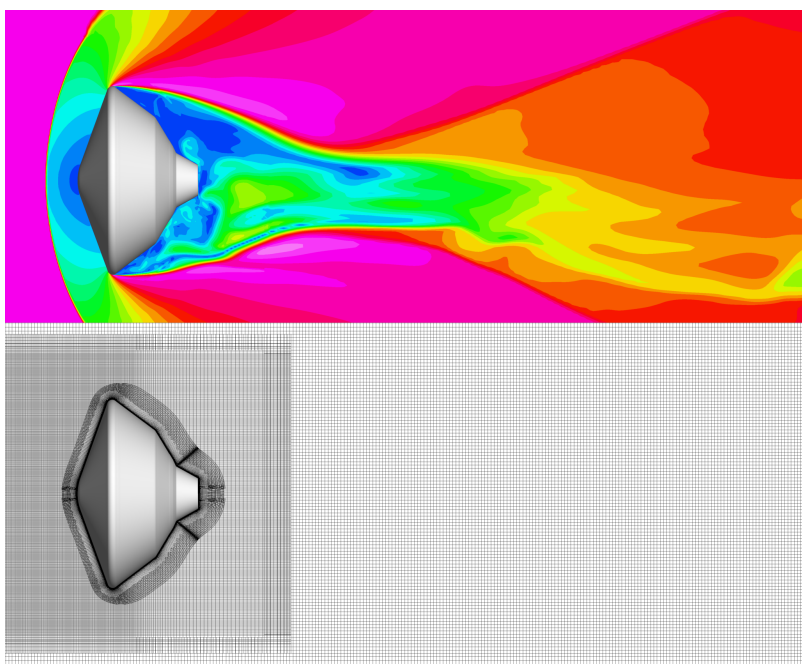

(a) $\alpha=0^{\circ}$

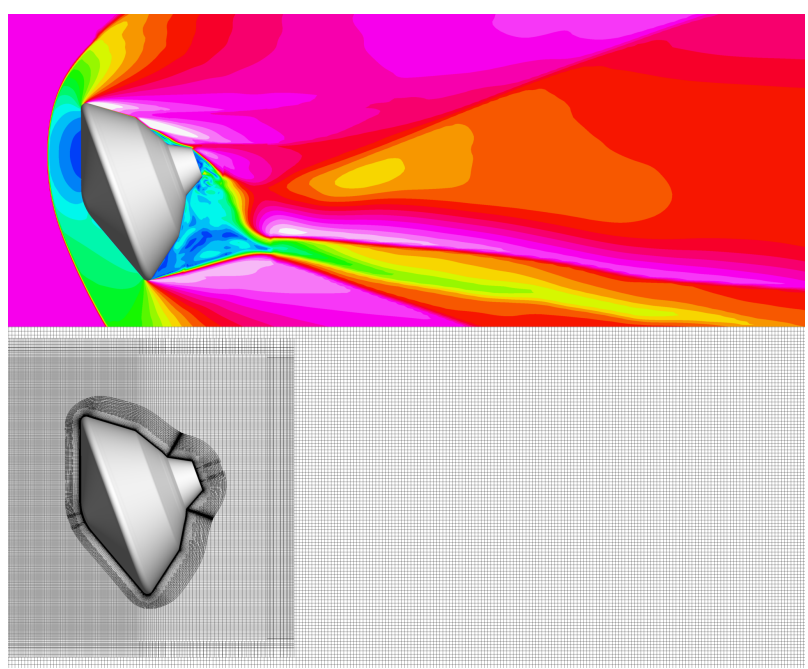

(b) $\alpha=20^{\circ}$

Figure 6. Grid and Mach number for dynamic simulation with $30^{\circ}$ initial pitch amplitude at two instances in time.

\section{Static Aerodynamic Coefficients}

It is useful, before looking at the integrated aerodynamic coefficients, to briefly discuss some features of the flowfield for this geometry at these conditions. Figure 7a shows instantaneous contours of Mach in a pitch plane slice for a US3D simulation at Mach 3.0 and $\alpha=30^{\circ}$, while fig. $7 \mathrm{~b}$ shows the backshell pressure distribution for the same. An important feature of this geometry at this high angle of attack is that the flow is attached on the first conic section of windward side of the backshell. This is especially evident in the pressure contours where it manifests as a higher pressure on this section. Furthermore, that attachment coupled with the expansion provided by the second backshell conic, results in a high-speed shear layer that 
impinges on the third conic section, resulting in another high pressure region. It should be noted that, in the case of the attachment on the first conic, the integrated effect of the resultant high-pressure region creates a moment that works against the restorative moment provided by the forebody.

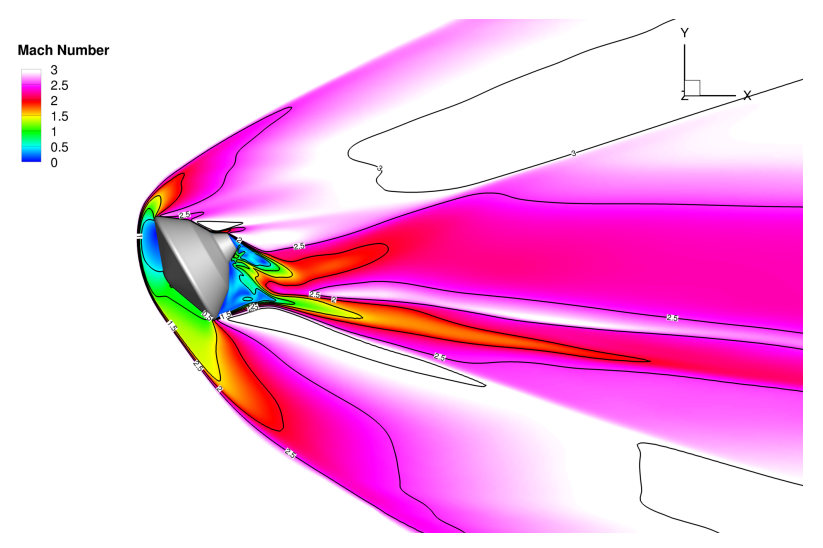

(a) Pitch plane Mach contours

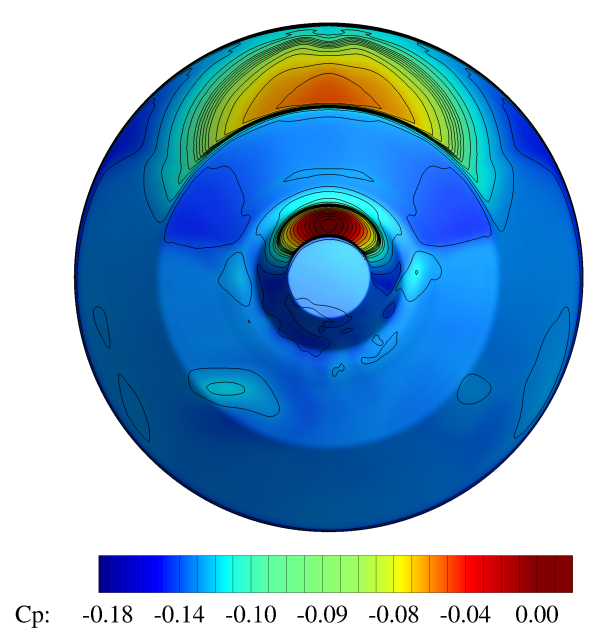

(b) Backshell pressure coefficient

Figure 7. Visualizations for Mach 3.0 and $\alpha=30^{\circ}$.

By contrast, at low angle of attack, the flow in and around the backshell is completely separated. Figure 8 shows instantaneous contours of the capsule again at Mach 3.0, but now at $\alpha=0^{\circ}$. We see in this case a larger wake with a weaker closure. The flow appears to detach from the surface at the apex of the capsule shoulder. Looking at the backshell pressure distribution, we see a comparatively uniform distribution.

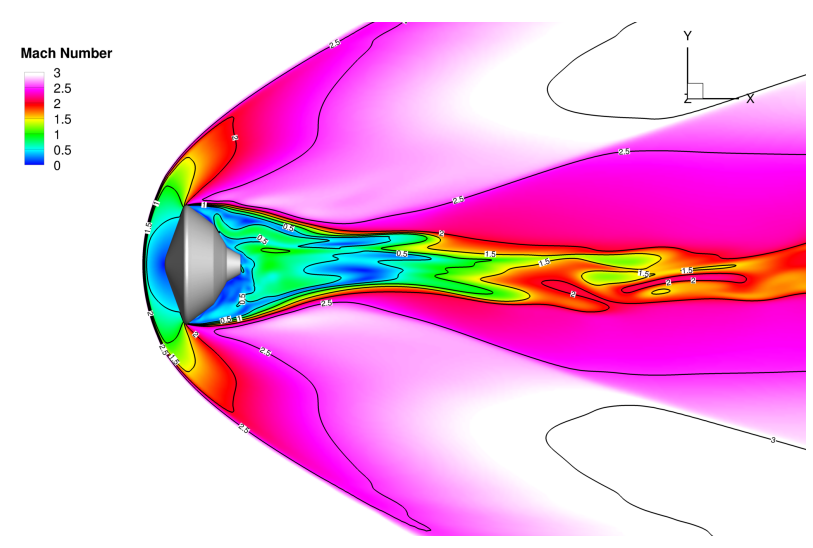

(a) Pitch plane Mach contours

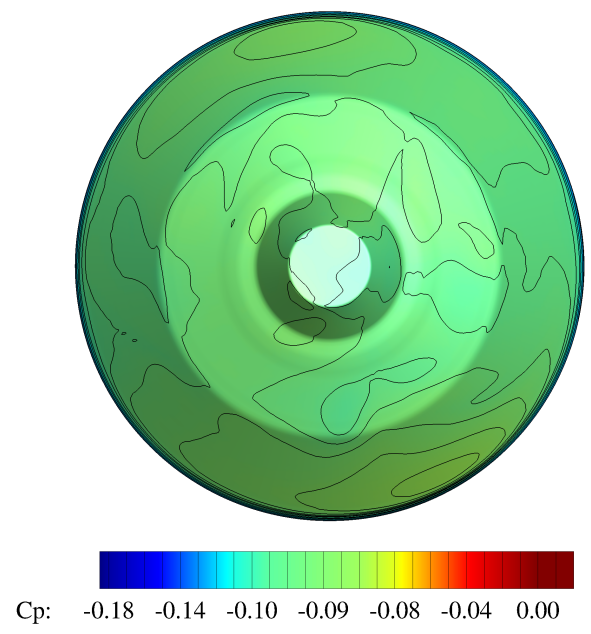

(b) Backshell pressure coefficient

Figure 8. Visualizations for Mach 3.0 and $\alpha=0^{\circ}$.

For the static analysis, we first compare predicted static aerodynamic coefficients for each of the flow solvers. Figure 9 shows the computed axial force coefficient, $C_{A}$, and pitching moment coefficient, $C_{m}$, for Mach 1.5, 2.0, and 3.0, and $\alpha$ ranging from $0^{\circ}$ to $30^{\circ}$. The same grid system was used for all computations; in the case of US3D the capsule was rotated and the mesh deformed around it, while for OVERFLOW the mesh attached to the capsule was rotated against the same background mesh. 


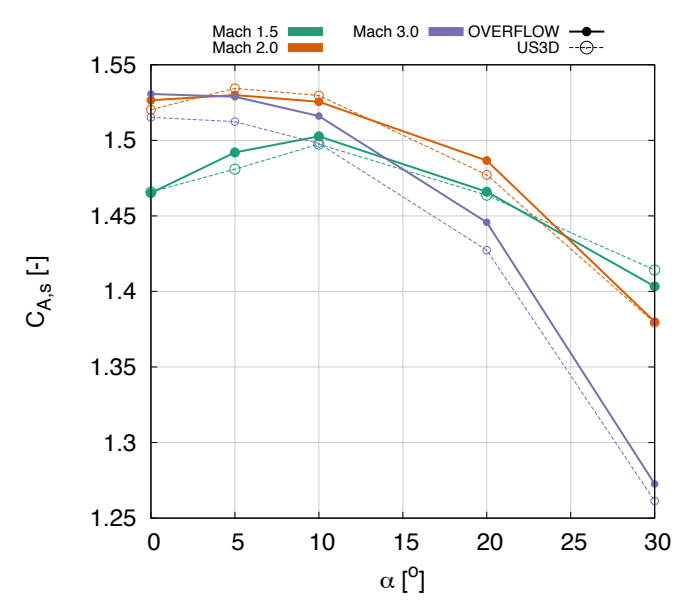

(a) Axial force coefficient, $C_{A}$

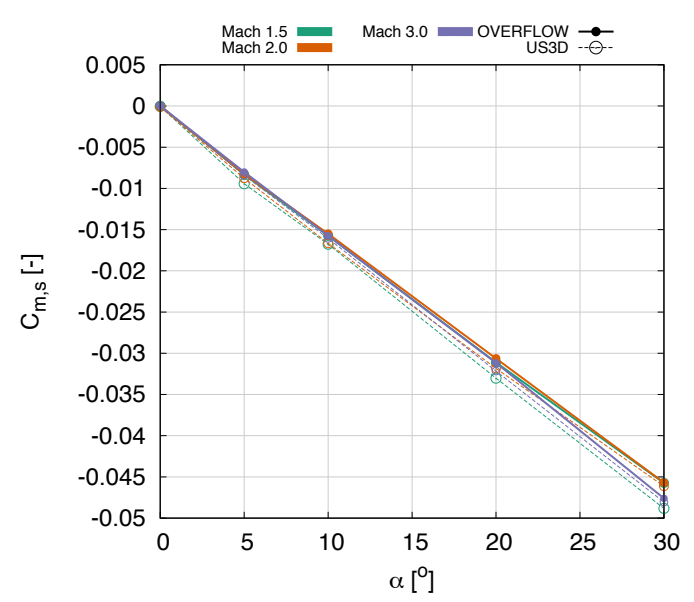

(b) Pitching moment coefficient, $C_{m}$

Figure 9. Computed static aerodynamic coefficients.

For the most part, in Figures 9(a) and 9(b), we see good agreement in the computed static aerodynamics. There are differences. They are worth highlighting before moving on to the dynamic results as they will manifest themselves in the dynamics. First, in Fig. 9(a), we see that US3D tends to predict a slightly lower value for the coefficient for most cases. This is the result of a generally higher predicted base pressure in the case of US3D. For the pitching moment coefficient, we see that US3D predicts a slightly steeper slope to this curve. This has an impact, which is seen in the predicted frequencies of pitch oscillation. Finally, it is worth noting that both codes predict a "dip" in the axial force coefficient at low angle of attack $\left(<5^{\circ}\right)$ at Mach 1.5. Although, there is not static wind tunnel data for this particular model, this is not typical of experimental results from similar capsule shapes. It is not currently clear if this result should be considered anomalous; however it may be important to note that the CFD simulations were of course run without the presence of a sting in the wake, and that at Mach 1.5 sting effects for an experiment could be expected to be significant.

\section{Dynamic Analysis}

The primary focus of the current work is to compare and assess the capabilities of OVERFLOW and US3D for performing dynamic simulations of entry vehicles. At the time of this writing the data from the ballistic range experiment is not yet available for comparison; therefore, for the purposes of this preliminary assessment, we have prescribed a run matrix which bounds the expected range of Mach numbers and initial amplitudes. The run matrix for the dynamic analysis can be seen in Tab. 2 .

\begin{tabular}{c|ccccc}
\hline Case & $M_{\infty}$ & $\alpha_{\text {init }}\left[^{\circ}\right]$ & $V_{\infty}[\mathrm{m} / \mathrm{s}]$ & $R_{D}\left[\times 10^{-} 6\right]$ & DoF's \\
\hline 1 & 3.0 & 30 & 1020.79 & 6.50 & free-to-pitch \\
2 & $\cdot$ & 5 & $\cdot$ & $\cdot$ & $\cdot$ \\
3 & 1.5 & 30 & 510.39 & 3.25 & $\cdot$ \\
4 & $\cdot$ & 5 & $\cdot$ & $\cdot$ & $\cdot$ \\
5 & 3.0 & 30 & 1020.79 & 6.50 & lifting and decelerating \\
\hline
\end{tabular}

Table 2. Simulation run matrix for the dynamic analysis.

We have selected the parameters such that they bound the range of interest for dynamic stability of entry capsules. Above Mach 3.0 there is typically sufficient damping in the pitch oscillations to allow for nominal parachute deploy conditions. Below Mach 1.5, and indeed probably sooner, Heritage sphere-cone shapes are 
known to become dynamically unstable, with potentially unacceptable oscillations in the pitch angle. This, as stated in the introduction, necessitates parachute deployment at higher Mach numbers, and thus poses engineering challenges, as well as imposes constraints on the entry, descent, and landing (EDL) sequence.

For this code-to-code comparison, we have also chosen to constrain the motion to 1 degree-of-freedom (DoF) for the 4 oscillating cases. The capsule for these simulations is allowed to pitch freely due to the aerodynamic moments, while the "yaw" and "roll" motions are suppressed. This simplification allows for more straightforward comparison, as well as data reduction. Case 5, in this table, is

Finally, for these cases, the inflow conditions are held constant for a given trajectory (i.e. there is no deceleration, lifting, or sideslip). The freestream conditions correspond to standard atmospheric conditions at sea level $\left(T_{\infty}=288 \mathrm{~K}, \rho_{\infty}=1.225 \mathrm{~kg} / \mathrm{m}^{3}\right)$, as this is likely to be representative of the conditions at the ballistic range at Aberdeen. The free-stream velocity, $V_{\infty}$, for each is provided in Tab. 2 .

The initialization procedure for each case, and for each code, was to first utilize the respective mesh motion approach detailed in Sec. IV to pitch the capsule to prescribed pitch amplitude for the given case. Then, the simulation is run statically until the integrated loads are statistically converged. The solution is then restarted with the code's respective rigid body dynamics solver turned on, and the vehicle is allowed to rotate under the influence of aerodynamic loading. It should be noted that this initialization procedure results in an initial condition for the dynamic trajectory that is strictly not physical, as the fluid dynamic environment around the capsule - in particular, the wake - is not realistic compared to that in an experiment or flight where the flight article is continuously oscillating. It is assumed that the effect of this strictly unphysical initial condition is dissipated as solution time progresses, however rigorous quantification of this effect is left to future work. For each case, we have run the simulation out to approximately 3 oscillation cycles. We output the following data as function of time: the pitch angle $\alpha$, the integrated aerodynamic forces and moments including the separate contributions of the forebody and backshell to the total pitching moment, and the surface pressure at discrete locations along the body. Each of these data will be discussed individually in subsequent sections.

\section{A. Pitch Angle Trajectories}

The first simulation outputs that we compare are the pitch angle trajectories predicted by both codes. Figure 10 shows the predicted pitch angle $\alpha$ vs. time predicted by the two codes. Figure 10(a) show the trajectories for Case 1 having an initial amplitude of $30^{\circ}$, while Fig. 10(b) shows the trajectories for Case 2 having an initial amplitude of $5^{\circ}$. We see in these figures that both codes predict damped pitch oscillations for both initial amplitudes, with the degree of damping - consistent with intuition - appearing to be greater for the larger amplitude case.

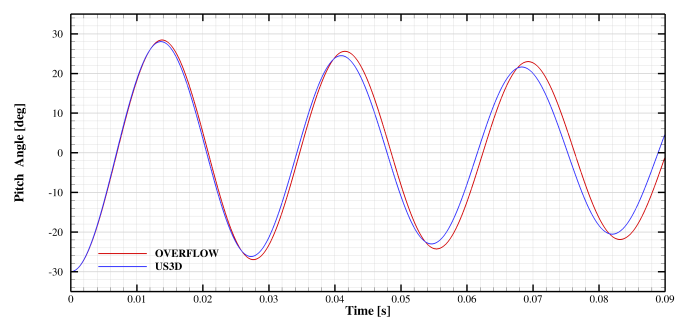

(a) High amplitude (initial $\alpha=30^{\circ}$ )

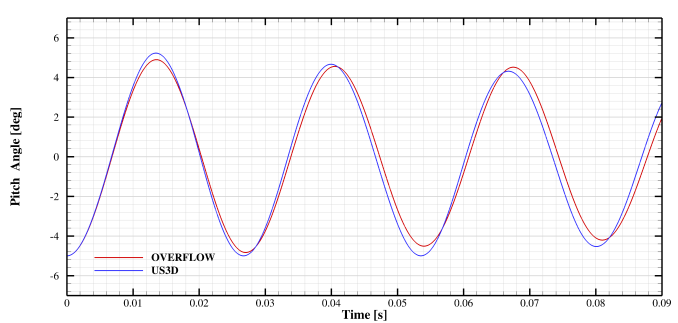

(b) Low amplitude (initial $\alpha=5^{\circ}$ )

Figure 10. Comparison of trajectories for Mach 3.0.

Comparing the predictions of the two codes, they agree fairly well for these cases. The most notable difference is the slight offset in the frequency. In this case - and in all cases that follow - the US3D solver predicts a slightly higher frequency than OVERFLOW. The frequency of oscillation for ballistic capsule is largely a function of the pitching moment slope, $C_{m \alpha}$, and the inertial properties of the model. A "steeper" $C_{m_{\alpha}}$ results in a higher frequency (analogous to the spring constant in a simple harmonic oscillator). Therefore we expect US3D to predict a slightly higher frequency due to the observed small difference in $C_{m}$ seen in Fig. 9b. Computed "effective" frequencies for each code and case can be found appendix B, Fig. 26; they are omitted here for the sake of brevity. The observed discrepancy in frequency corresponds to an 
approximate $1.5 \%$ difference in mean frequency between the two predictions. It is also worth noting that US3D appears to predict somewhat more damping for the higher amplitude oscillation, while predicting the somewhat less damping for the low amplitude trajectory. This will be further quantified in a subsequent section where we examine the predicted dynamic derivatives.

Figure 11 shows the computed trajectories for the two Mach 1.5 cases for both the $30^{\circ}$, and $5^{\circ}$ initial amplitudes. For the high initial amplitude case, we observe similar behavior as in case 1, with both codes predicting a damped pitch oscillation, though for this case the damping appears slightly less pronounced. Again, we observe a slightly higher frequency for the US3D results as compared to that from OVERFLOW, with the discrepancy - $\approx 2.5 \%$ of the mean - being more pronounced than for the higher Mach cases. For the low amplitude case (Fig.11(b)) we note that - as opposed to the other cases presented - both codes predict undamped pitch oscillation growth. Furthermore, we observe that there appears to be noticeably less damping for the computed US3D trajectory. We shall attribute these differences to differences in the backshell pressure, which will be examined in greater detail in a subsequent section.

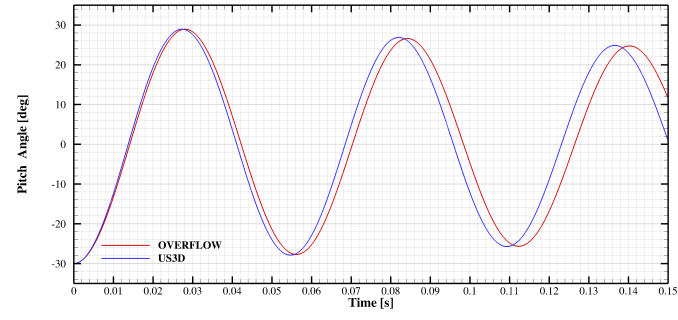

(a) High amplitude (initial $\alpha=30^{\circ}$ )

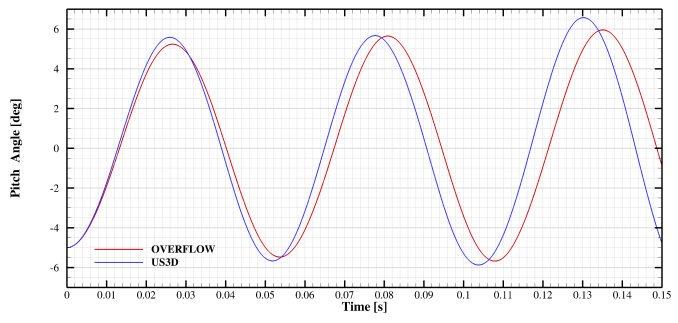

(b) Low amplitude (initial $\alpha=5^{\circ}$ )

Figure 11. Comparison of trajectories for Mach 1.5.

\section{B. Static and Dynamic Derivatives}

One of the objectives from this work is to calculate the dynamic aerodynamic coefficients. CFD provides a large volume of data for dynamics analysis with force, moment, and vehicle attitudes know for all time. This allows for detailed analysis of the range of cases considered here in order to develop an aerodynamic model for the pitch-only capsule flight. While the approach used here differs from what will be used to reduce the ballistic range data, it is identical between the two solvers. This will serve to illustrate the differences and similarities between predicted aerodynamic coefficients - both dynamic and static - derived from the dynamic simulations, but may not provide good comparison to experimentally derived quantities when those become available. The procedure used to reduce the data is described in detail in appendix A.

We begin by looking at the computed coefficients for Case 1, which can be seen in Fig.12. Figure 12(a) shows both the pitching moment coefficient derived from the static calculations, as described in Sec.V, as well as that derived from the dynamic simulations. Here, we observe very good agreement between the two codes, as well as between the statically and dynamically derived coefficients. Again, we see a very slightly steeper pitching moment slope for US3D.

For the pitch damping coefficient, $C_{m q}$ we see similar shape to the curve between codes, however the magnitudes are different. Here, OVERFLOW predicts a greater (less negative) $C_{m q}$ as compared with US3D, which is consistent with the observation from the previous section that the US3D trajectory appeared more damped, as well as consistent with the observation that both codes produced damped (negative $C_{m_{q}}$ ) trajectories for Case 1.

Figure 13 shows the computed coefficients for Case 2. Here, we find very good agreement for the pitching moment coefficient. For the pitch damping coefficient, we see that now US3D predicts a more positive $C_{m q}$ than OVERFLOW. This is consistent with the observation of the trajectory from the previous section.

Looking now at the computed coefficients for Mach 1.5, Fig.14 shows the results for Case 3. We find again good agreement for the pitching moment coefficient, although we note some deviation at high angle of attack. Consistent with previous observations, both codes produce negative $C_{m q}$ curves. The pitch damping coefficient shows some disagreement as in previous cases, however in this case, US3D has less damping at 


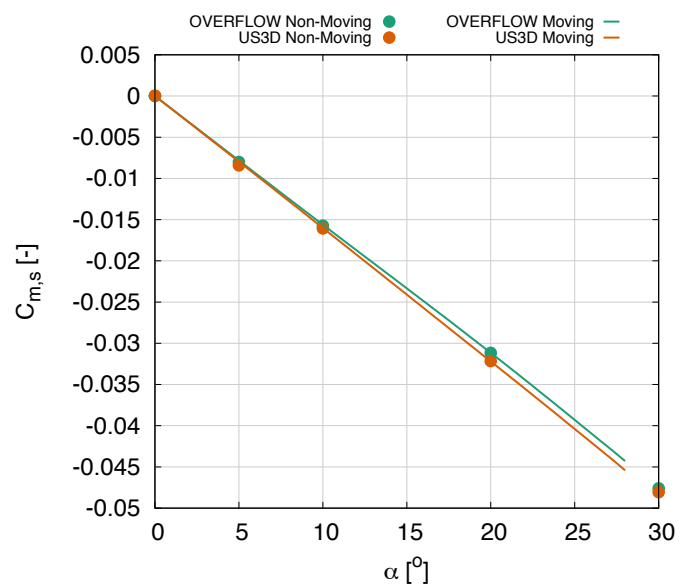

(a) Statically and dynamically derived moment coefficient, $C_{m}$

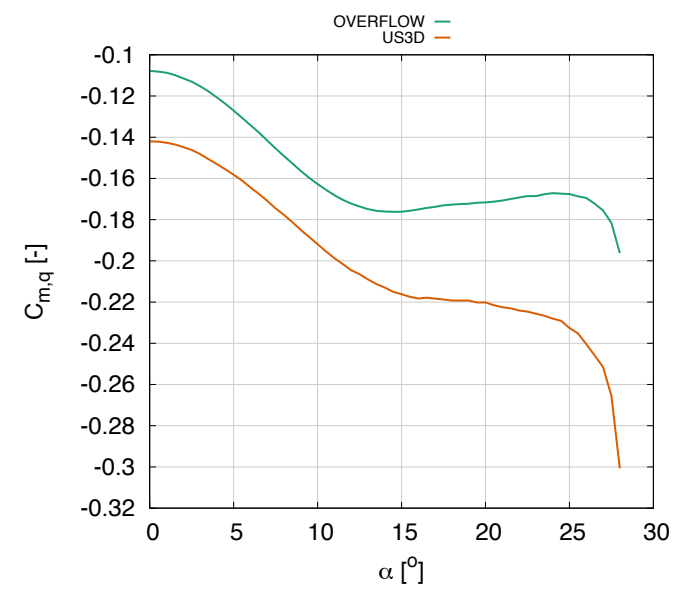

(b) Pitch damping coefficient, $C_{m, q}$

Figure 12. Static and dynamic derivatives for Case 1.

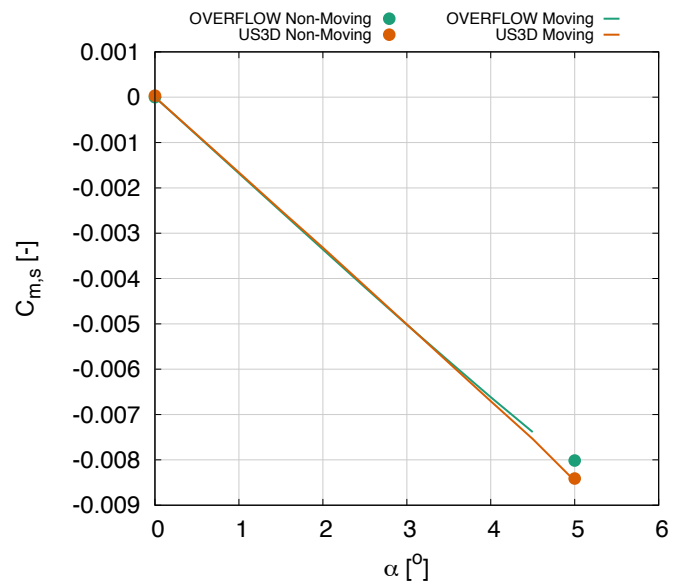

(a) Statically and dynamically derived moment coefficient, $C_{m}$

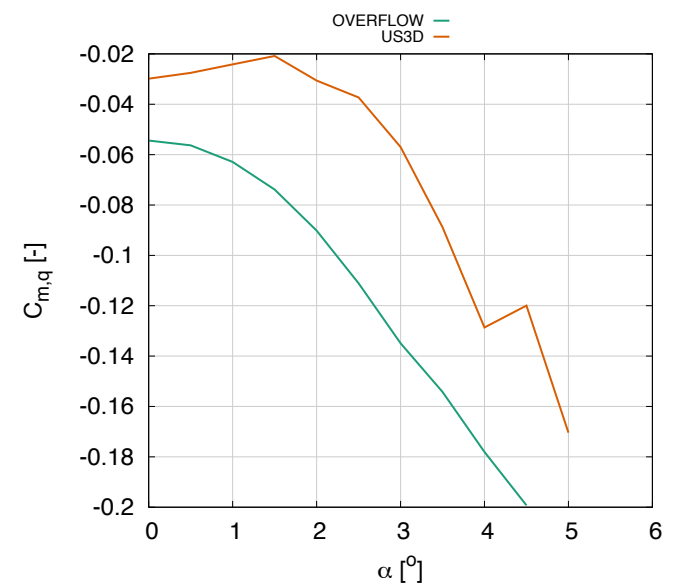

(b) Pitch damping coefficient, $C_{m, q}$

Figure 13. Static and dynamic derivatives for Case 2. 
low pitch angle, and more damping at high pitch angle. It is difficult to infer a conclusion from this result it may be the case that more oscillations are needed to obtain a more meaningful curve for this case.

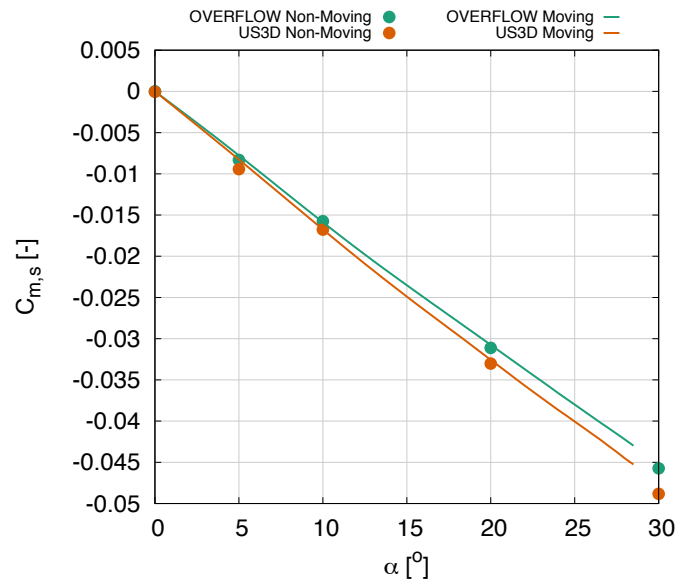

(a) Statically and dynamically derived moment coefficient, $C_{m}$

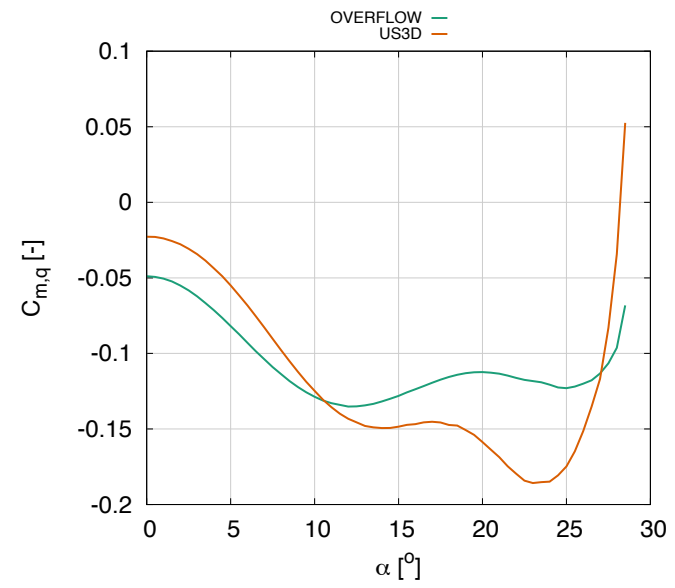

(b) Pitch damping coefficient, $C_{m, q}$

Figure 14. Static and dynamic derivatives for Case 3.

Finally, looking at Case 4, we again find good agreement between the solvers for the pitching moment coefficient. Comparing computed pitch damping coefficients we see that both have positive pitch damping at low amplitude. This is consistent with the observation that the computed trajectories for case 4 show growth in pitch oscillation amplitude. Additionally, we note that US3D here produces a larger magnitude for the pitch damping coefficient.

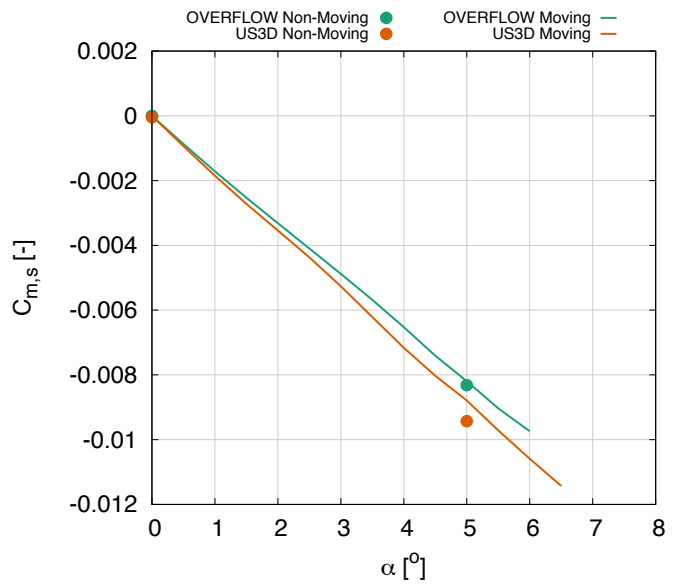

(a) Statically and dynamically derived moment coefficient, $C_{m}$

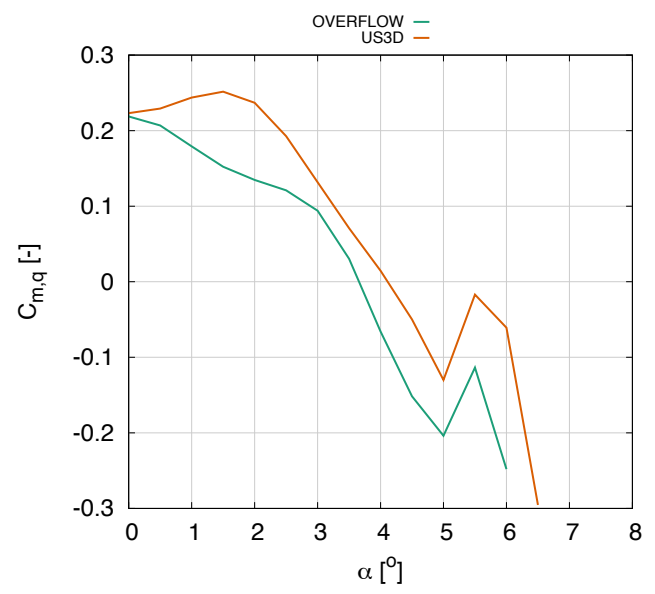

(b) Pitch damping coefficient, $C_{m, q}$

Figure 15. Static and dynamic derivatives for Case 4.

\section{Forebody and Backshell Moments}

To gain more insight into the observed phenomena and quantities from the previous sections, we now look at the evolution of the pitching moment. More specifically, we decompose the total integrated pitching moment into the separate contribution from the forebody and the backshell of the capsule. Here, the forebody is defined as the surface from the nose to the apex of the shoulder, and the backshell is defined as everything aft of the the apex of the shoulder. Note that it does not strictly correspond to the heatshield and the backshell of the flight vehicle, where the heatshield wraps around the shoulder past the apex. 
For these comparisons, we plot the two codes on separate sets of axes for clarity. On each plot, the backshell moment is plotted in solid lines against the left axis, while the forebody moment is plotted in dashed lines against the right axis. Figure 16 shows the decomposed moments for case 1 . The first thing to note in both plots is that the backshell contribution to the moment is small compared to that of the forebody, on the order of $1-5 \%$. This is because of the high Mach number and resultant high dynamic pressure. Under such circumstances, the integrated loads on the forebody will dominate effects from the comparatively low pressure wake. The forebody moment for sphere-cone aeroshells such as this tends to to damp pitch oscillations, and thus for this case we see damped pitch angle trajectories for both codes.

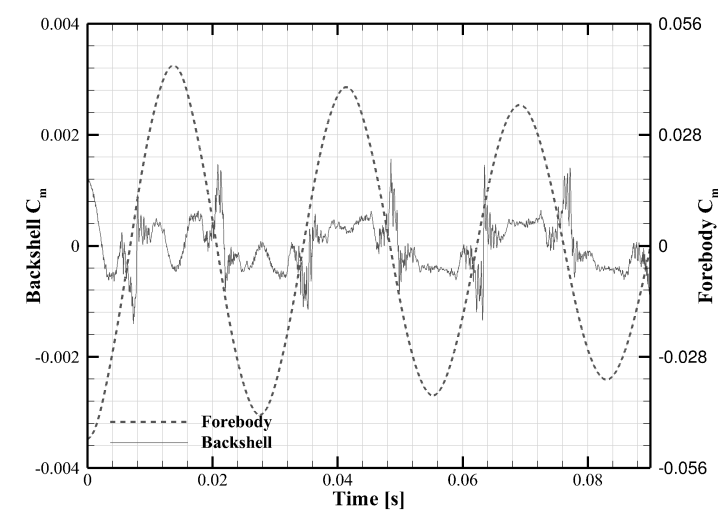

(a) OVERFLOW

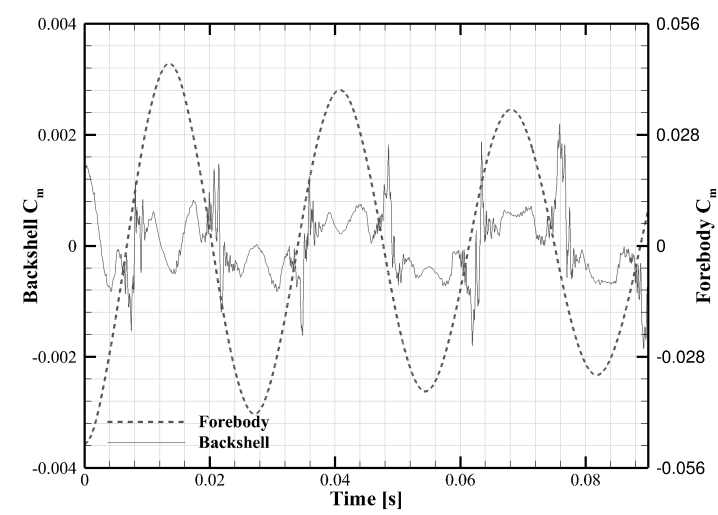

(b) US3D

Figure 16. Decomposed forebody and backshell moments for case 1.

In Fig. 17 we present a zoomed-in comparison of the first cycle of the moment history for Case 1 to better illustrate a couple additional features of this dataset. The first thing to note is that the magnitude of the US3D backshell moment is slightly larger at the initialization. We further observe that the backshell moment magnitude for both codes is much larger at the initialization than during the trajectory. This may be in part due to the damping in the pitch amplitude, but also likely illustrates the problematic nature of the initialization procedure. It may be the case that this backshell pressure environment would not be attainable during "natural" dynamic motion of the model, but rather is a byproduct of the static initialization.

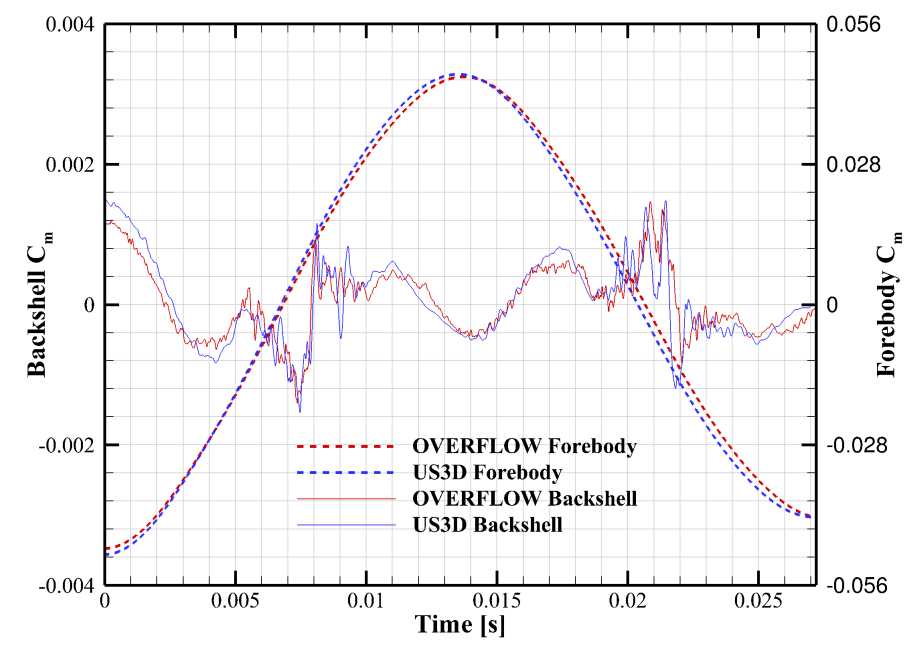

Figure 17. First pitch oscillation cycle of the Case 1 decomposed forebody and backshell moments.

Finally, it is interesting to note the complex dynamics of the backshell moment for this condition. The 
flow attachment and separation dynamics are readily reflected in this trace. At initialization, the flow on the windward side of the backshell is attached (see Fig.7) resulting in higher pressure on this side. For this case, this results in an integrated backshell moment which opposes the forebody. This effect is most pronounced at the initialization, but can be seen again later in the trajectory $(t \approx 0.015 s)$. In principle, this would be destabilizing, however, as discussed above, the forebody damping overwhelms the influence of the wake for this condition.

For a contrasting example, we look to the dynamic moments for Case 4. Recall from Sec. VI. A that for this case we observe an unstable trajectory predicted by both codes.The US3D solution is the less stable of the two. For insight into why these conditions result in an unstable trajectory, as well as why there is some difference in the degree of predicted instability, we can look to the decomposed moments as plotted in Fig. 18.

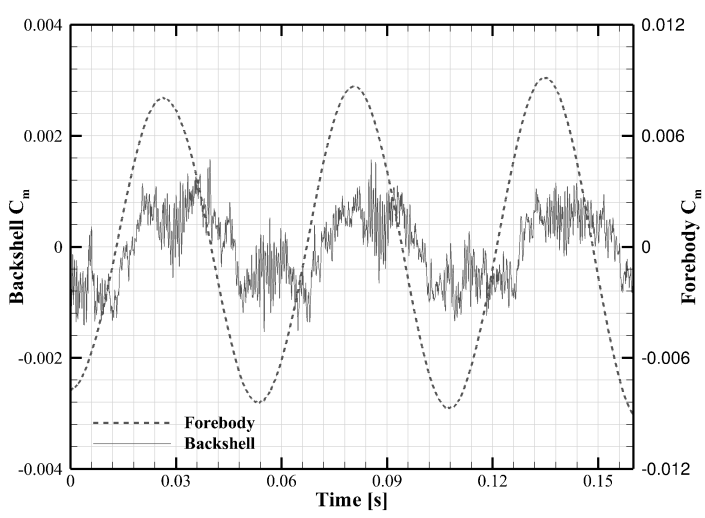

(a) OVERFLOW

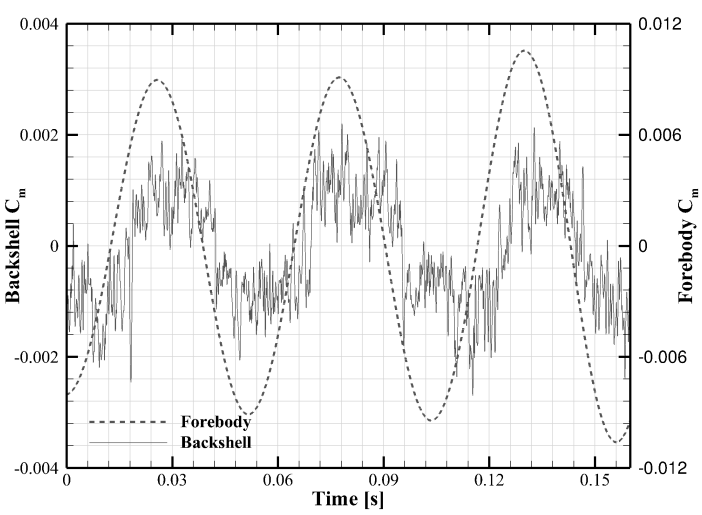

(b) US3D

Figure 18. Decomposed forebody and backshell moments for case 4 .

In contrast to the previously analyzed case, we note that the magnitude of the backshell moment is a greater percentage of the forebody moment compared with the previous example $(\approx 15 \%)$. Therefore, at this lower Mach number, the influence of the wake on the dynamics of the entry vehicle is likely to be more significant. Additionally, for both codes, we can observe that there is a "lag" of the response of the aftbody moment to the pitch angle of the model, whereas it can be assumed that the forebody moment responds effectively instantaneously. This "phase lag" phenomenon has been cited as one of the mechanisms of dynamic instability in entry capsules. For a more detailed treatment of this theory, the reader is referred to the work of Abe et al., ${ }^{3}$ Teramoto et al., ${ }^{4}$ and Kazemba et al. ${ }^{5}$ For the current analysis it is sufficient to say that, based on this theory, the greater the phase offset, and the greater the amplitude of the backshell moment, the greater will be the instability. Comparing the two plots, we do not see significant difference in the extent of phase lag between the two codes. There is however a difference in the magnitude of the backshell moment contribution, with the US3D simulation producing a larger backshell moment at the peaks. It was observed previously that the US3D trajectory produced more growth in pitch amplitude than did the the OVERFLOW simulation. We conclude that increased amplitude growth observed in the US3D trajectory is the result of this increased magnitude in contribution from the backshell moment.

\section{Surface Pressure Data}

The final analysis that we present here are surface pressures at discrete locations on the body. Pressure probe locations selected for this analysis can be seen in Fig. 19, and the approximate locations of these probes in a coordinate system, whose origin lies at the vehicle nose, is shown in Tab. 3. Note that all pressure probes reside in the pitch plane, which corresponds with $z=0.0 \mathrm{~cm}$ in current frame of reference.

The probe array consists of six surface locations; two on the forebody, and four on the backshell. The locations for these simulated pressure probes are arbitrary, but are likely to correspond roughly to candidate locations for the experimental article. For each case presented here, the probes are initially on the windward side prior to being released to dynamically oscillate. We use the probes here to provide further insight into 


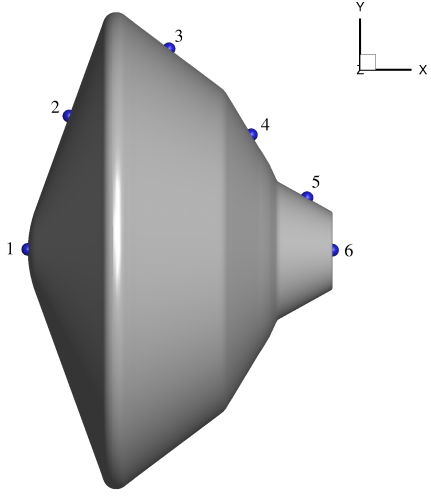

(a) Side view

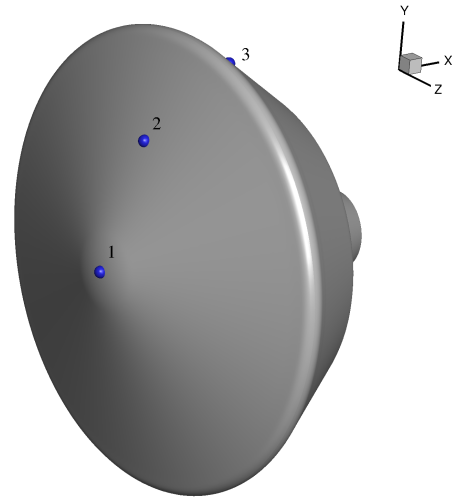

(b) Front view

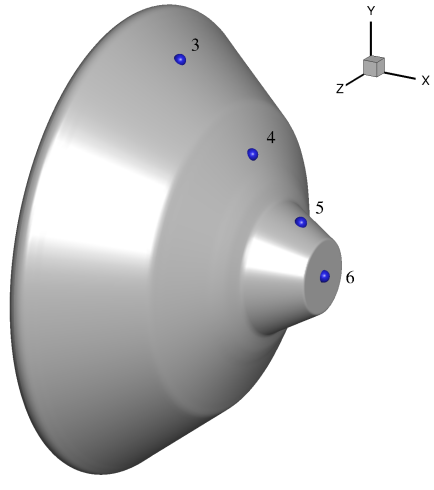

(c) Aft view

Figure 19. Surface pressure probes.

\begin{tabular}{c|cc}
\hline Probe & $x[\mathrm{~cm}]$ & $y[\mathrm{~cm}]$ \\
\hline 1 & 0.00 & 0.00 \\
2 & 0.77 & 2.52 \\
3 & 2.67 & 3.79 \\
4 & 4.22 & 2.18 \\
5 & 5.26 & 0.98 \\
6 & 5.75 & 0.00 \\
\hline
\end{tabular}

Table 3. Approximate locations for the pressure measurements.

the wake dynamics that are driving the backshell moments discussed in the previous section, as well as further interrogate the differences between the two solvers and their computed trajectories.

For the current work, and for the sake of brevity, we focus the analysis on results from case 1. Pressure data from the remaining cases are provided in appendix C. Pressure data here is reported as a nondimensional pressure coefficient $\left(C_{P}=\left(p-p_{\infty}\right) / \frac{1}{2} \rho V_{\infty}^{2}\right)$.

Figure 20 shows the output for the six simulated pressure probes for case 1 . Note that in these figures the top two plots show data from the forebody, while the remaining four are from the backshell. In the forebody data, we observe smooth pressure traces for the full trajectory, as expected, given that the flow here is steady and attached. We also note that there is a slight difference in the pressure at the apex location at $\alpha=0^{\circ}$ (the peaks in these plots). The decay in the minima is due to the damping of the pitch amplitude, as previously discussed. Additionally, we see a "flat" profile on the forebody flank pressure probe when it is on the windward side.

Looking now at the backshell pressure data (probes $3-6$ ), we observe more unsteady character, as well as more complex coupling to the dynamics. First, in probe 3, we are able to see the aforementioned dynamic separation and reattachment of the flow. The attachment is evident in portions of the trace where the pressure becomes smooth, followed by a low-pressure undershoot, and then flow separation. Both codes produce similar general features for this plot; however we see notably lower pressures for the OVERFLOW result at low pitch angle where the flow is expected to be separated and unsteady. This is for the most part consistent across all of the backshell surface pressure traces; when the flow is separated, US3D tends to predict higher pressures than does OVERFLOW. This conclusion provides some explanation for the observed differences in integrated quantities discussed in previous sections.

Another notable difference between the two codes can be seen in the data from probe 5 , on the third conic segment of the backshell. At these conditions and pitch angle, there is a strong impingement by the high speed shear layer at the second conic segment. This results in an observed spike in the pressure at this location at high angle of attack. Notable is that here, OVERFLOW predicts a significantly higher peak for this spike than does US3D. Furthermore, the amplitude of the spike in the US3D result appears to decay more 


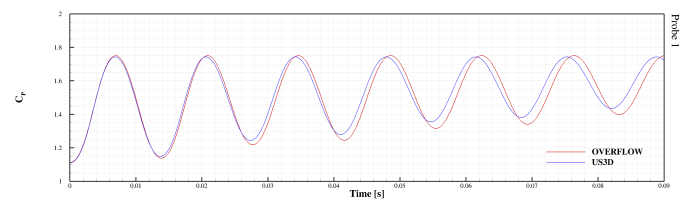

(a) Probe 1

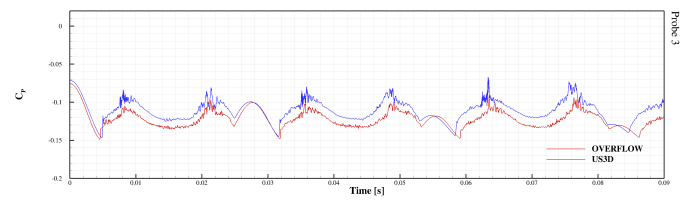

(c) Probe 3

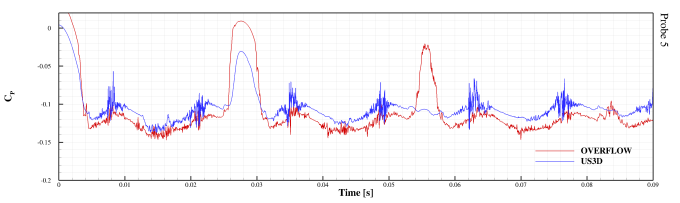

(e) Probe 5

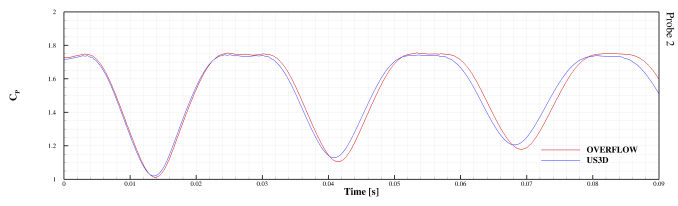

(b) Probe 2

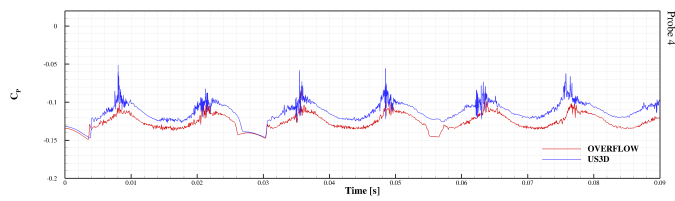

(d) Probe 4

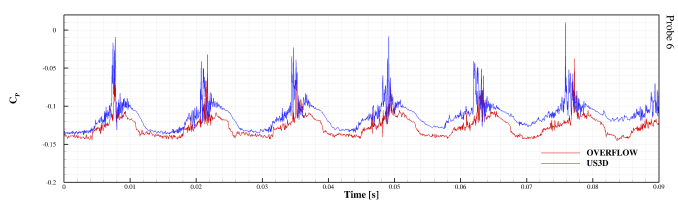

(f) Probe 6

Figure 20. Backshell pressure probe comparsons for case 1.

rapidly than does the OVERFLOW result. It was noted in a previous section that this case was the only one where OVERFLOW predicted less damping than US3D. This observed difference in the predicted pressure may explain this, as the increase in pressure at this location tends to augment the backshell component of the pitching moment.

\section{E. Downrange and Vertical Displacement}

\section{Conclusions}

Dynamic CFD analysis was performed on a notional ballistic range model using two different flow solvers, OVERFLOW and US3D. Overall, the two solvers produced similar results, with some key differences. To summarize:

- Both codes predicted damped pitch oscillations for all cases except for case 4 (low Mach, low amplitude), where both codes predicted growth in the pitch angle.

- In all cases, US3D predicted a slightly higher frequency of pitch oscillation. We attribute this to the slightly higher pitching moment slope, $C_{m \alpha}$, as compared with OVERFLOW.

- In all but case 1 , OVERFLOW predicted greater damping. This is reflected in both the trajectories themselves, as well as in the derived pitch damping coefficients.

- Larger amplitude in the backshell contribution to the pitching moment for US3D is a likely culprit for the observed reduction in damping in the pitch oscillations as compared with the OVERFLOW results.

- Where the flow is separated, OVERFLOW tends to predict backshell surface pressures that are lower than US3D.

We infer from these conclusions that differences in the predicted characteristics of the separated wake result in the differences in the predicted pitch damping performance of the model. Factors, both numerical and physical, that may influence the wake could include different spatial and temporal discretizations, turbulence models, and/or grid resolution. Investigations into the sensitivity of the dynamic simulations to these factors is the subject of on-going work. 


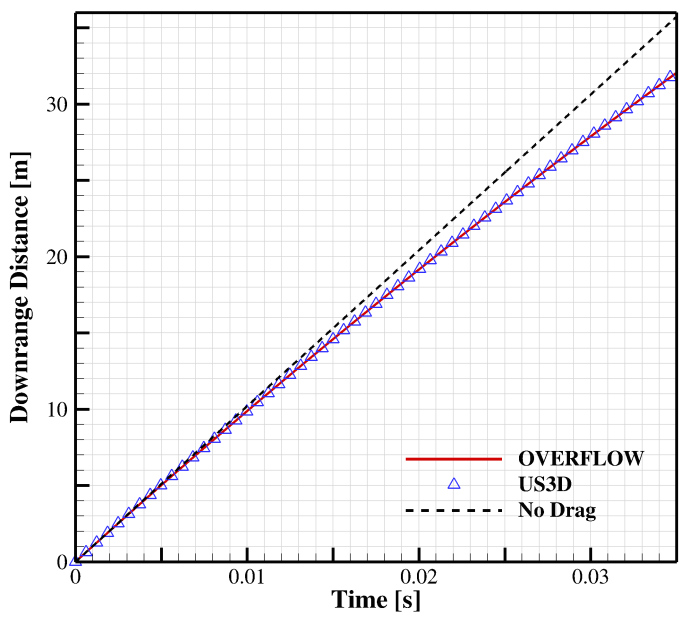

(a) Downrange distance

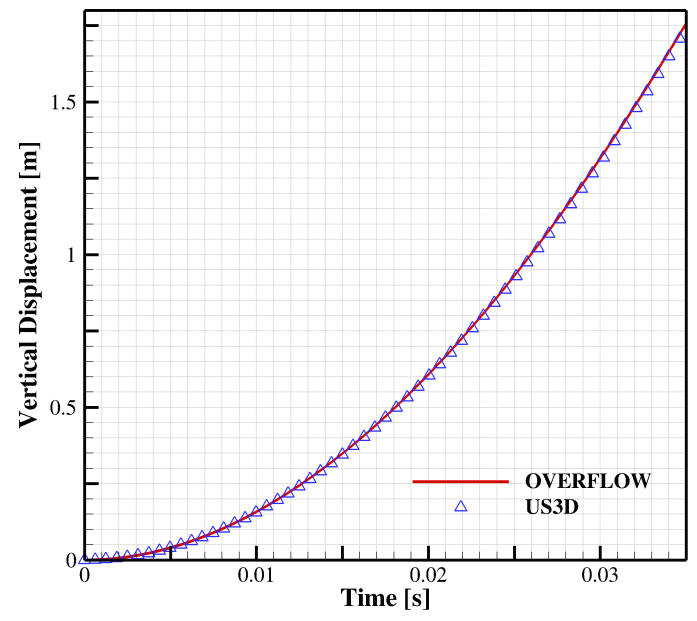

(b) Vertical displacement

Figure 21. Translational displacements for Case 5.

\section{Future Work}

At the time of submission for the current work, the ballistic range experiments upon which these simulations were based have been performed, but the data from these experiments have not yet been processed. Future work will be focused on running simulations at the reconstructed conditions from the experiment, and comparing trajectories and surface pressures to the data gathered from the range. Additionally, as it was concluded that differences in the predicted wake environment have a notable effect on the aerodynamic performance of the model in the simulation, additional analysis will be done to further characterize the influence of numerics and physical modeling choices on this behavior.

\section{Acknowledgments}

This work is supported through the NASA Engineering and Safety Center (NESC) Early Career Engineer Program. Additional support provided by the Entry Systems Modeling (ESM) project within NASA's Game Changing Development Program. Mr. Brock is supported through NASA contract NNA15BB15C to AMA Inc.. The authors gratefully acknowledge Dr. Joseph Schulz and Dr. Michael Barnhardt for there helpful comments on the manuscript.

\section{References}

\footnotetext{
${ }^{1}$ Karlgaard, C. D., Kutty, P., Schoenenberger, M., Munk, M. M., Little, A., Kuhl, C. A., and Shidner, J., "Mars Science Laboratory Entry Atmospheric Data System Trajectory and Atmosphere Reconstruction," Journal of Spacecraft and Rockets, Vol. 51, No. 4, July 2014, pp. 1029-1047.

${ }^{2}$ Schoenenberger, M., Norman, J. V., Karlgaard, C., Kutty, P., and Way, D., "Assessment of the Reconstructed Aerodynamics of the Mars Science Laboratory Entry Vehicle," Journal of Spacecraft and Rockets, Vol. 51, No. 4, July 2014, pp. 1076-1093.

${ }^{3}$ Abe, T., Sato, S., Matsukawa, Y., Yamamoto, K., and Hiraoka, K., "Study for Dynamically Unstable Motion of Reentry Capsule," Tech. rep., Feb. 2003.

${ }^{4}$ Teramoto, S., Hiraki, K., and Fujii, K., "Numerical Analysis of Dynamic Stability of a Reentry Capsule at Transonic Speeds," AIAA Journal, Vol. 39, No. 4, April 2001, pp. 1-8.

${ }^{5}$ Kazemba, C. D., Braun, R. D., Schoenenberger, M., and Clark, I. G., "Dynamic Stability Analysis of Blunt-Body Entry Vehicles Using Time-Lagged Aftbody Pitching Moments," Journal of Spacecraft and Rockets, Vol. 52, No. 2, March 2015, pp. 393-403.

${ }^{6}$ Schoenenberger, M., Yates, L., and Hathaway, W., "Dynamic Stability Testing of the Mars Science Laboratory Entry Capsule," 41st AIAA Thermophysics Conference, American Institute of Aeronautics and Astronautics, Reston, Virigina, June 2012 .
} 
${ }^{7}$ Nompelis, I., Drayna, T. W., and Candler, G. V., "Development of a Hybrid Unstructured Implicit Solver for the Simulation of Reacting Flows Over Complex Geometries," 34th AIAA Fluid Dynamics Conference, The University of Minnesota, Portland, July 2004, pp. 1-12.

${ }^{8}$ Nompelis, I., Drayna, T. W., and Candler, G. V., "A Parallel Unstructured Implicit Solver for Hypersonic Reacting Flow Simulation," 17th AIAA Computational Fluid Dynamics Conference, The University of Minnesota, Toronto, July 2005, pp. $1-17$.

${ }^{9}$ Wright, M. J., Candler, G. V., and Bose, D., "Data-Parallel Line Relaxation Method for the Navier-Stokes Equations," AIAA Journal, Vol. 36, No. 9, Sept. 1998.

${ }^{10}$ Subbareddy, P. K. and Candler, G. V., "A fully discrete, kinetic energy consistent finite-volume scheme for compressible flows," Journal of Computational Physics, Vol. 228, No. 5, March 2009, pp. 1347-1364.

${ }^{11}$ Brock, J. M., Subbareddy, P. K., and Candler, G. V., "Detached-Eddy Simulations of Hypersonic Capsule Wake Flow," AIAA Journal, Nov. 2014, pp. 1-11.

${ }^{12}$ Rajagopalan, K. and Eiseman, P. R., "Automatic nested refinement: a technique for the generation of high quality multi-block structured grids for multi-scale problems using GridPro." Eng. Comput. (), Vol. 21, No. 1, 2005, pp. 29-35.

${ }^{13}$ Candler, G. V. and MacCormack, R. W., "Computation of weakly ionized hypersonic flows in thermochemical nonequilibrium," Journal of Thermophysics and Heat Transfer, Vol. 5, No. 3, July 1991, pp. 266-273.

${ }^{14}$ Hirsch, C., Numerical Computation of Internal and External Flows, Computational Methods for Inviscid and Viscous Flows, Wiley, Jan. 1991.

${ }^{15}$ Wright, M. J., A Family of Data-Parallel Relaxation Methods for the Navier-Stokes Equations, Ph.D. thesis, University of Minnesota, June 1997.

${ }^{16}$ Spalart, P. R., Jou, W.-h., Strelets, M., and Allmaras, S. R., "Comments on the Feasibility of LES for Wings, and on a Hybrid RANS/LES Approach," First AFOSR International Conference on DNS/LES, 1997.

${ }^{17}$ Spalart, P. and Allmaris, S., "A one-equation turbulence model for aerodynamic flows," 30th Aerospace Sciences Meeting and Exhibit, American Institute of Aeronautics and Astronautics, Reno, Nevada, June 1992, pp. 1-58.

${ }^{18}$ Catris, S. and Aupoix, B., "Density corrections for turbulence models," Aerospace Science and Technology, Vol. 4, No. 1, 2000, pp. 1-11.

${ }^{19}$ Nichols, R. H., Tramel, R. W., and Buning, P. G., "Solver and turbulence model upgrades to OVERFLOW 2 for unsteady and high-speed applications," AIAA Paper, 2006.

${ }^{20}$ Murman, S. M., Chan, W. M., Aftosmis, M. J., and Meakin, R. L., "An interface for specifying rigid-body motions for CFD applications," AIAA Paper, 2003.

${ }^{21}$ Chan, W. M., "The overgrid interface for computational simulations on overset grids," 32nd AIAA Fluid Dynamics Conference and Exhibit, American Institute of Aeronautics and Astronautics, St. Louis, Missouri, 2002.

${ }^{22}$ Chan, W. M., "Advances in Software Tools for Pre-processing and Post-processing of Overset Grid Computations," 9th International Conference on Numerical Grid Generation in Computational Field Simulations, 2005.

${ }^{23}$ Chan, W. M., Kim, N., and Pandya, S. A., "Advances in Domain Connectivity for Overset Grids Using the X-Rays Approach," 7th International Conference on Computational Fluid Dynamics, Big Island, Hawaii, July 2012.

${ }^{24}$ Tramel, R. W., Nichols, R. H., and Buning, P. G., "Addition of improved shock-capturing schemes to OVERFLOW 2.1," 32nd AIAA Fluid Dynamics Conference and Exhibit, San Antonio, Texas, 2009.

${ }^{25}$ Menter, F. R., "Two-equation eddy-viscosity turbulence models for engineering applications," AIAA Journal, Vol. 32, No. 8, Aug. 1994, pp. 1598-1605.

${ }^{26}$ Stern, E. C., Gidzak, V. M., and Candler, G. V., "Estimation of Dynamic Stability Coefficients for Aerodynamic Decelerators Using CFD," 30th AIAA Applied Aerodynamics Conference, University of Minnesota, New Orleans, June 2012, pp. $1-14$.

${ }^{27}$ Murman, S. M., "Dynamic Simulations of Atmospheric-Entry Capsules," Journal of Spacecraft and Rockets, Vol. 46, No. 4, July 2009, pp. 829-835. 


\section{Appendices}

Here, we provide detail on the data reduction approach used to derive the dynamic derivatives, as well as additional data from the simulations which was omitted from main body of the paper in the interest of brevity, but which may be of interest to the reader. Additionally, we have endeavored to this point to provide sufficient detail such that other researchers could duplicate this work. This additional data could then be used as additional comparison for those who wish to assess their own tools.

\section{A. Data reduction methodology}

For single degree of freedom simulations, a linear aerodynamic model was selected for reconstruction of each integrated force and moment coefficient $(\phi)$ as shown in Eq. 1. The total coefficient, $C_{\phi}$, is the sum a static and dynamic component. Static simulations for a capsule at fixed $\alpha$ could be used to determine $C_{\phi, s}$ which is a function of the angle of attack. The dynamic portion is also assumed to be a function of the angle of attack and the non-dimensional pitch rate, $\hat{q}\left(\hat{q}=\frac{q}{V_{\infty} D_{0}}\right)$. This must be determined by running dynamic simulations or tests and cannot be obtained from static data.

$$
C_{\phi}(\alpha, \hat{q})=\underbrace{C_{\phi, s}(\alpha)}_{\text {static }}+\underbrace{\frac{\partial C_{\phi}(\alpha)}{\partial \hat{q}} \times \hat{q}}_{\text {dynamic }}
$$

Changing notation slightly, the dynamic coefficient, $C_{\phi, q}$, is obtained by means of linear regression. Integrated results from a single dynamic simulation can be partitioned by $\alpha$ and fit to a line with independent parameter $\hat{q}$. Equation 2 presents an interpretation from the linear fit. The slope of the resulting line can be interpreted as the dynamic coefficient and the intercept as an estimate of the static coefficient (no pitch rate) from the dynamic simulation. Results for both the static and dynamic coefficient can be tabulated and returned as an aerodynamic model valid for the trajectory from the dynamic data. An important note is that the dynamic coefficient is assumed to be linear with respect to pitch rate. This is not strictly true, as will be apparent in the results below.

$$
C_{\phi}(\alpha, \hat{q})=\underbrace{C_{\phi, s}(\alpha)}_{\text {intercept }}+\underbrace{C_{\phi, q}(\alpha)}_{\text {slope }} \times \hat{q}
$$

The following will show an illustrated example of the analysis technique used for these problems. A similar presentation has been shown in the work of others. ${ }^{27}$ It focuses on using the OVERFLOW data for Case 1, a free-to-pitch simulation of the MSL capsule at Mach 3.0 with an initial amplitude of $30^{\circ}$. Analysis of the pitching moment coefficient will be highlighted in the detailed example.

Figure 22(a) shows pitching moment coefficient and $\alpha$ as a function of time. While not significant at this Mach number and initial amplitude, the computational results were filtered by using a low-pass filter at $200 \mathrm{~Hz}$. At a number of angles of attack have been identified with open and closed circular symbols and the pitching moment at those angles of attack are identified by the symbols on the plot. In practice, data was calculated at every $0.5^{\circ}$ of $\alpha$. The same pitching moment data are shown in Fig. 22(b), but are now plotted against $\hat{q}$. For each value of $\alpha$, a linear fit can be made to determine the static and dynamic aerodynamic coefficients (Eq. 2).

The quality of the linear fit should be improved by increasing the number of points used in the regression. Symmetries in the problem (constrained pitch motion, axisymmetric vehicle, and rotational center location) imply that the static and dynamic coefficients should be symmetric at positive and negative $\alpha$. Figure takes advantage of these symmetries to mirror data at negative $\alpha$ and increase the effective number of samples at positive $\alpha$. This requires negating $q$ and $C_{m}$ as well as $\alpha$. Data at $\alpha=0^{\circ}$ can similarly be augmented by mirroring across $q$ and $C_{m}$, but not $\alpha$. Finally, a linear fit can be made through the resulting collection of points and the slope and intercept recorded as $C_{\phi, s}(\alpha)$ and $C_{\phi, q}(\alpha)$ (Fig. 23(b)).

Figure 24 shows the resulting values for $C_{\phi, s}(\alpha)$ and $C_{\phi, q}(\alpha)$. On the left, the static coefficient determined from analysis of the dynamic data (line) agrees well with the static results from the non-moving mesh (symbols). This provides some validation of the results. The right figure shows the dynamic coefficient from OVERFLOW. This process was repeated for all other cases and with results from both solvers.

This paper will focus on pitching moment, but static and dynamic coefficients of axial and normal force were computed as well and are plotted in Fig. 25. The static values agree well between the moving and 


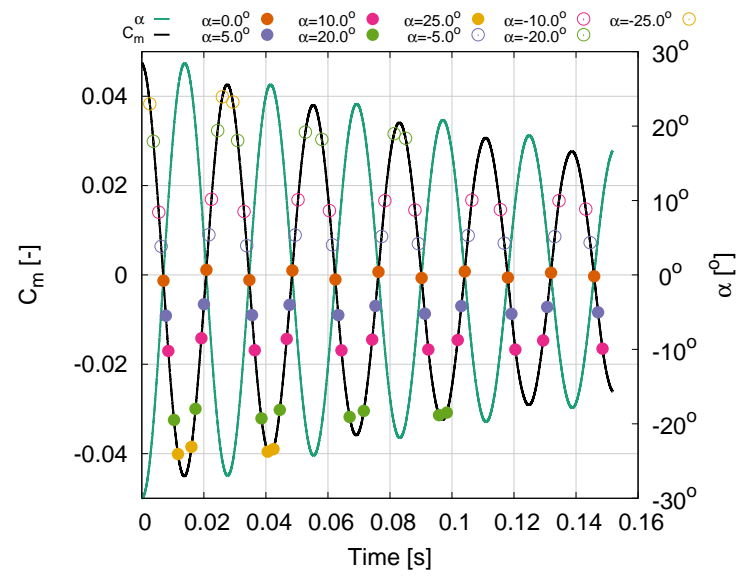

(a) Versus time

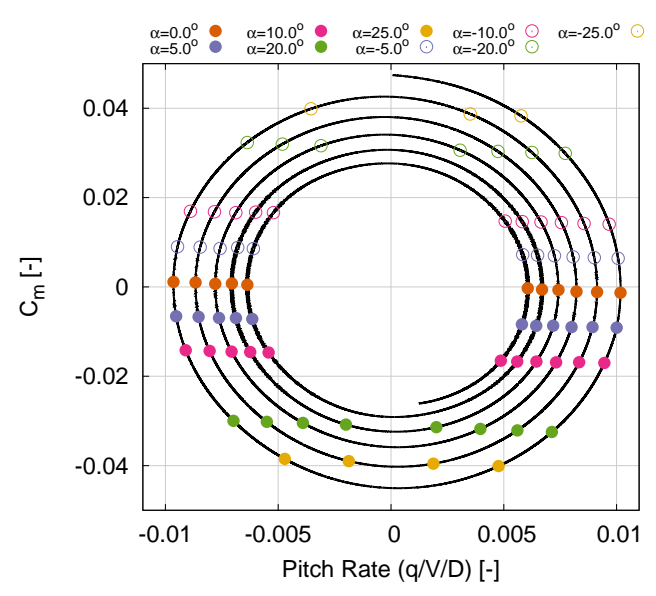

(b) Versus $\hat{q}$

Figure 22. Pitching moment coefficient history for Case 1 using OVERFLOW.

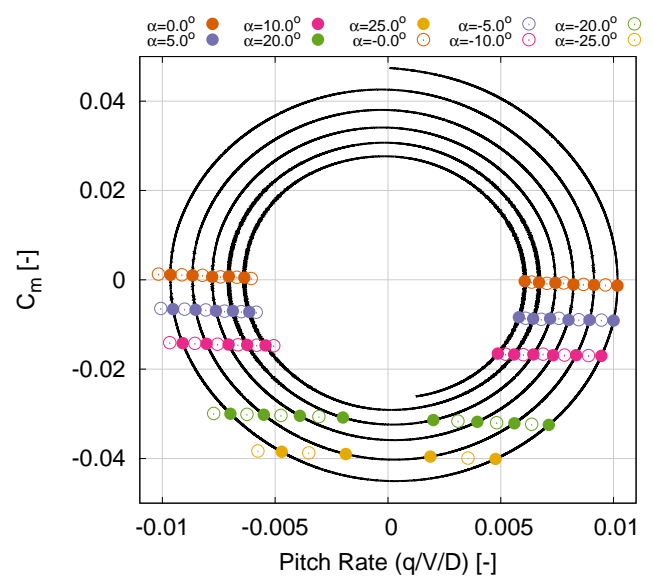

(a) Mirroring of negative $\alpha$ points

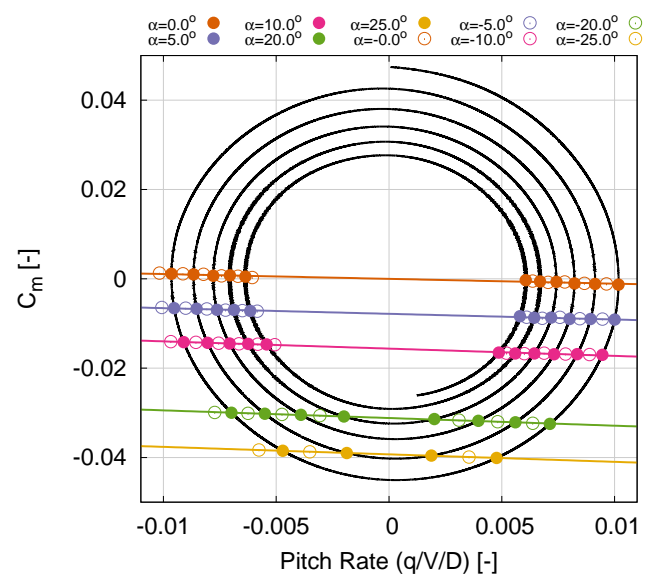

(b) Fit to available data

Figure 23. Intermediate steps in data analysis for OVERFLOW Case 1 data.

non-moving simulations. These coefficients are much less linear than $C_{m, s}$ and agreement is still good at this Mach number. 


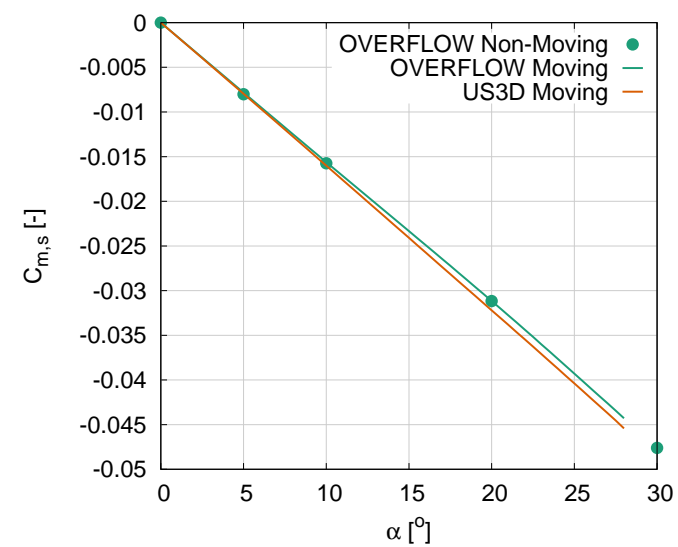

(a) Static coefficient

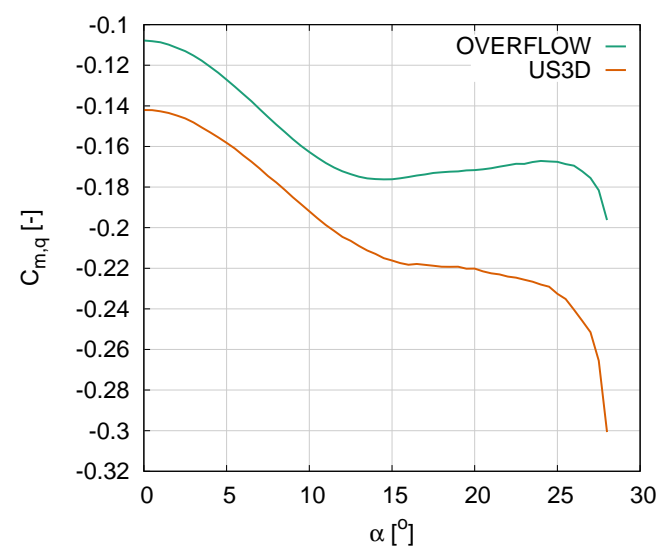

(b) Dynamic coefficient

Figure 24. Pitching moment coefficient results from Case 1.

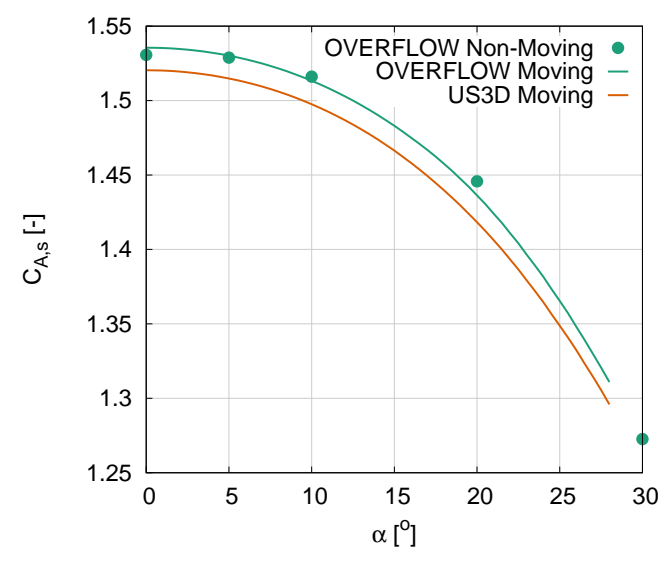

(a) Axial force coefficient

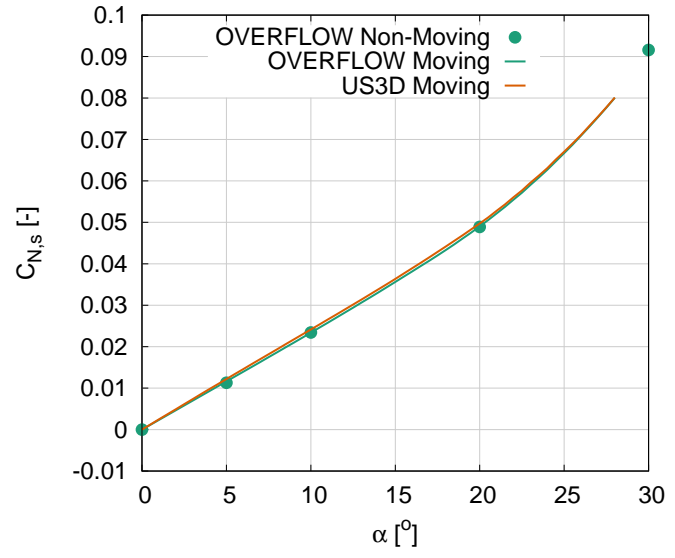

(b) Normal force coefficient

Figure 25. Static force coefficients from Case 1.

\section{B. Effective Frequencies}

These plots provide approximate predicted frequencies of oscillation for each of the cases and codes. Here the effective frequency is defined as the inverse of the peak-to-peak period, for a given trajectory segment. 


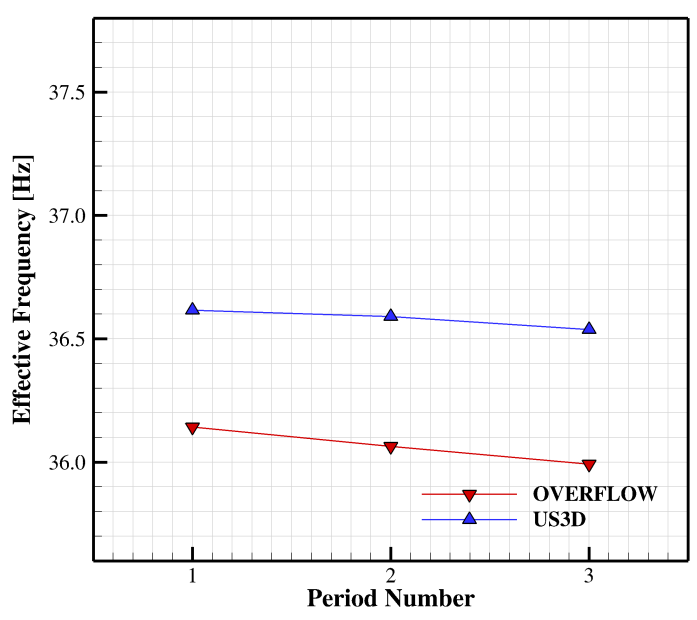

(a) Case 1

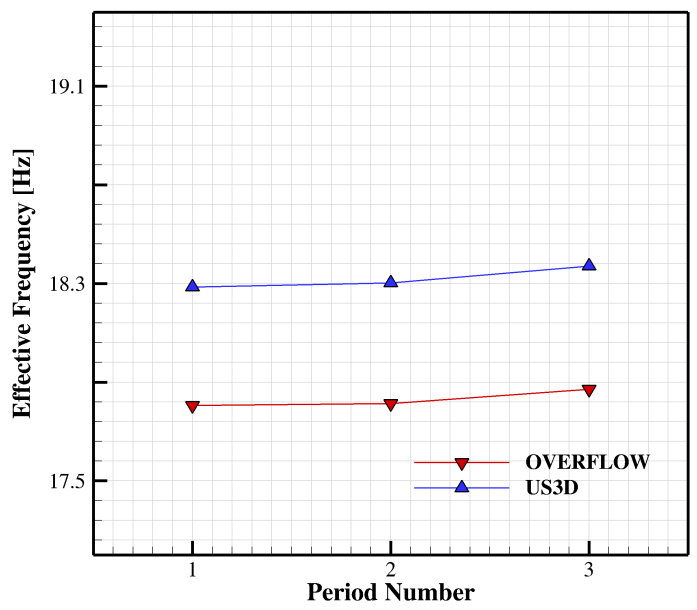

(c) Case 3

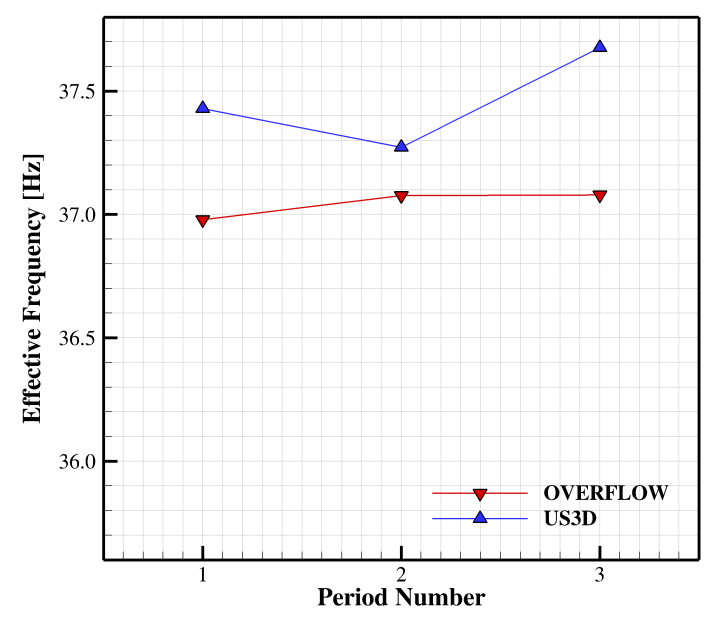

(b) Case 2

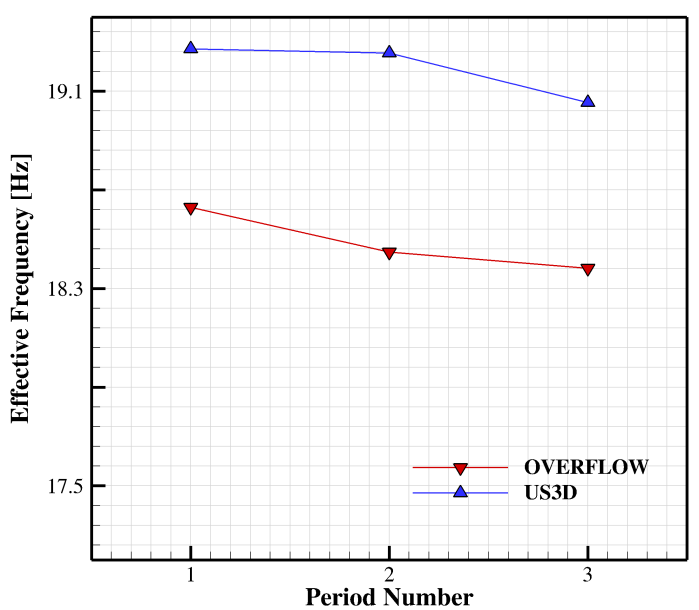

(d) Case 4

Figure 26. Comparison of effective frequencies for each period of oscillation, for each case. 


\section{Additional surface pressure data}

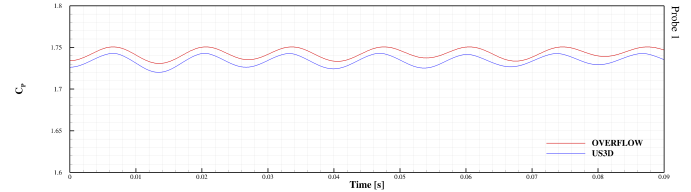

(a) Probe 1

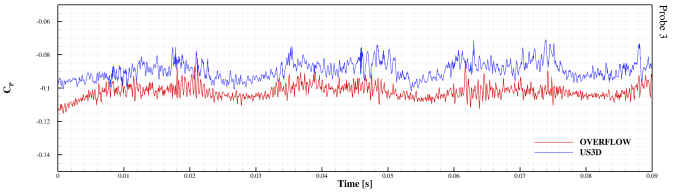

(c) Probe 3

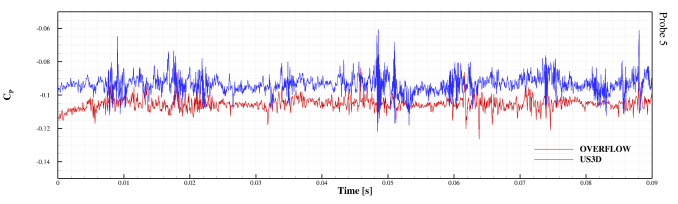

(e) Probe 5

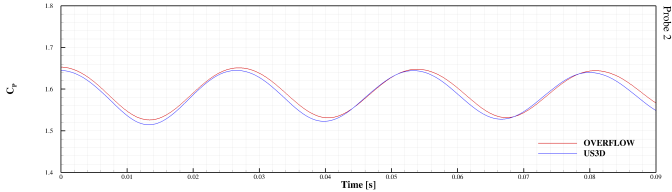

(b) Probe 2

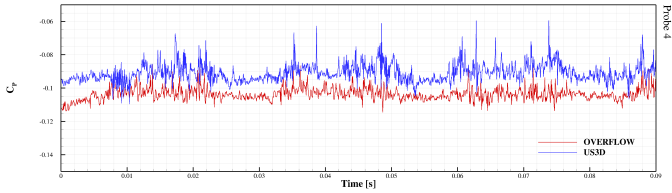

(d) Probe 4

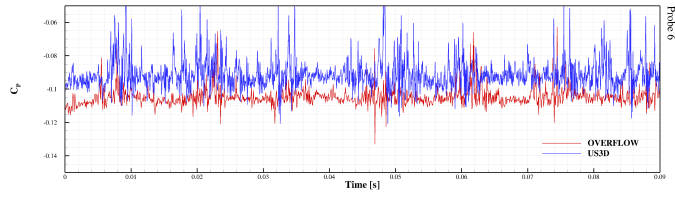

(f) Probe 6

Figure 27. Backshell pressure probe comparsons for case 2. 


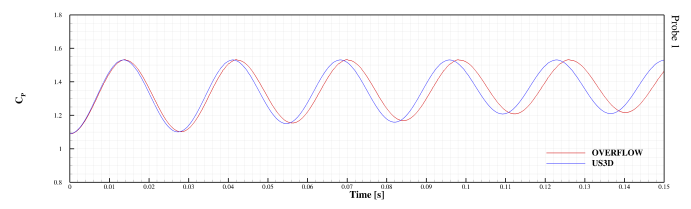

(a) Probe 1

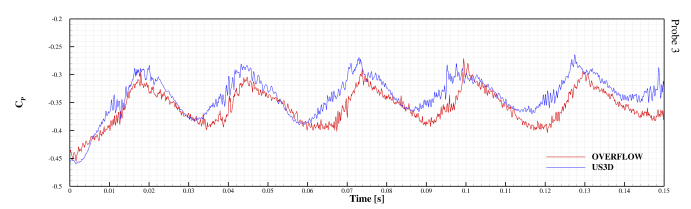

(c) Probe 3

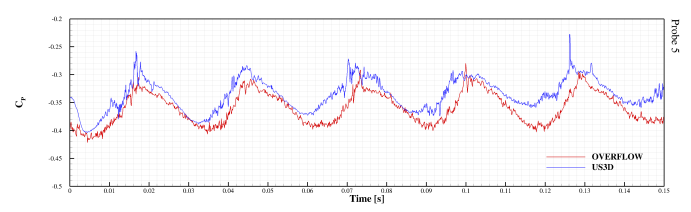

(e) Probe 5

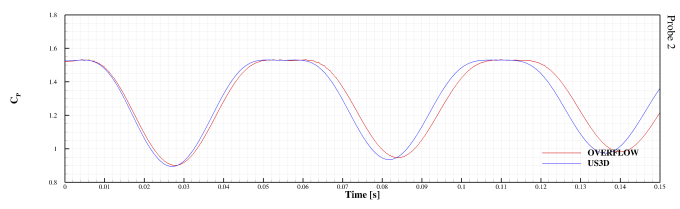

(b) Probe 2

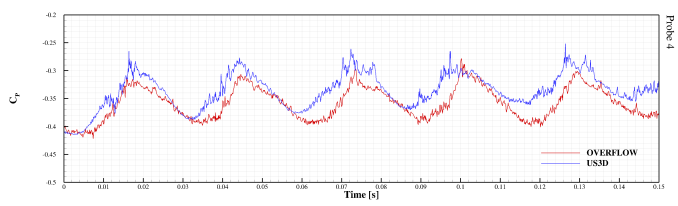

(d) Probe 4

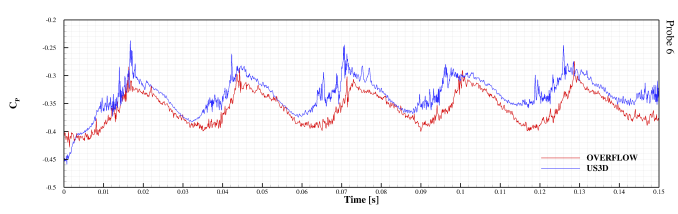

(f) Probe 6

Figure 28. Backshell pressure probe comparsons for case 3 .

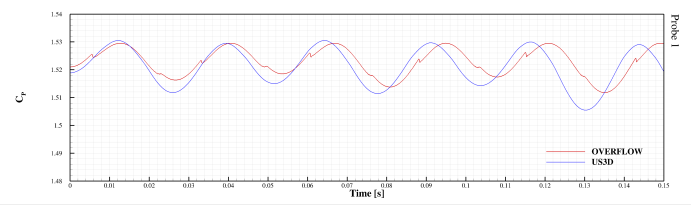

(a) Probe 1

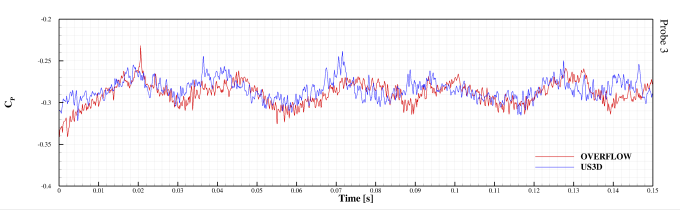

(c) Probe 3

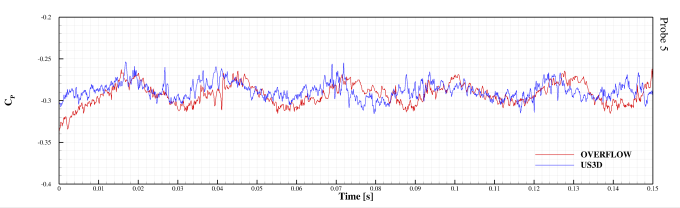

(e) Probe 5

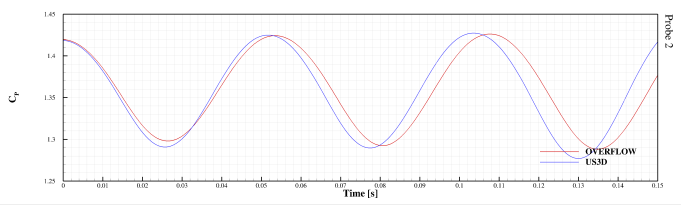

(b) Probe 2

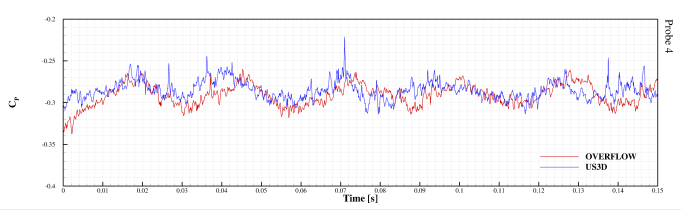

(d) Probe 4

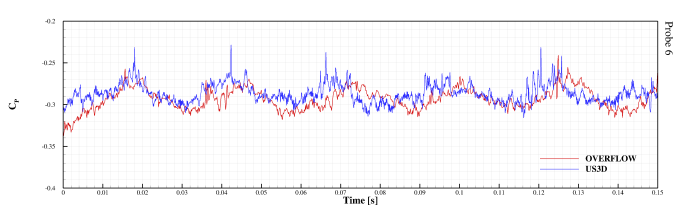

(f) Probe 6

Figure 29. Backshell pressure probe comparsons for case 4 . 
D. Flowfield Visualzations for Mach 1.5

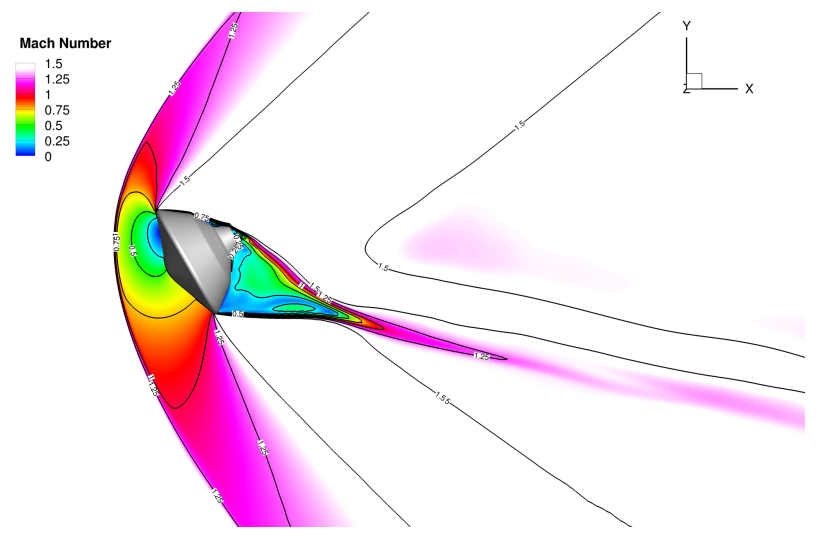

(a) Pitch plane Mach contours

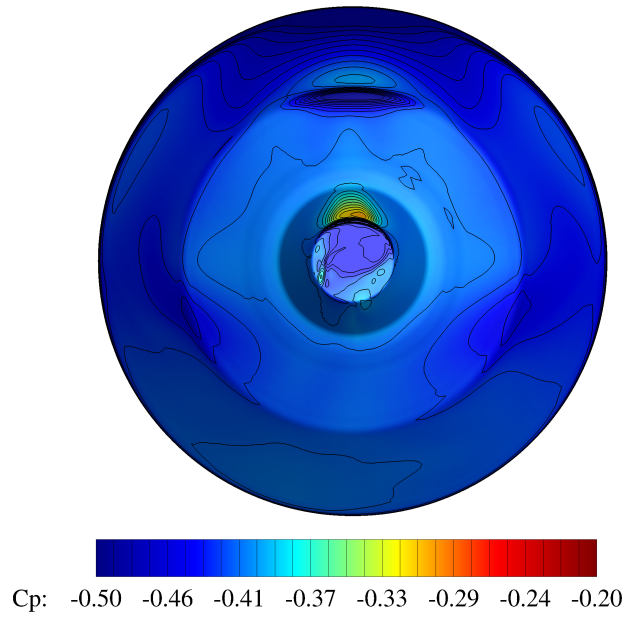

(b) Backshell pressure coefficient

Figure 30. Visualizations for Mach 1.5 and $\alpha=30^{\circ}$.

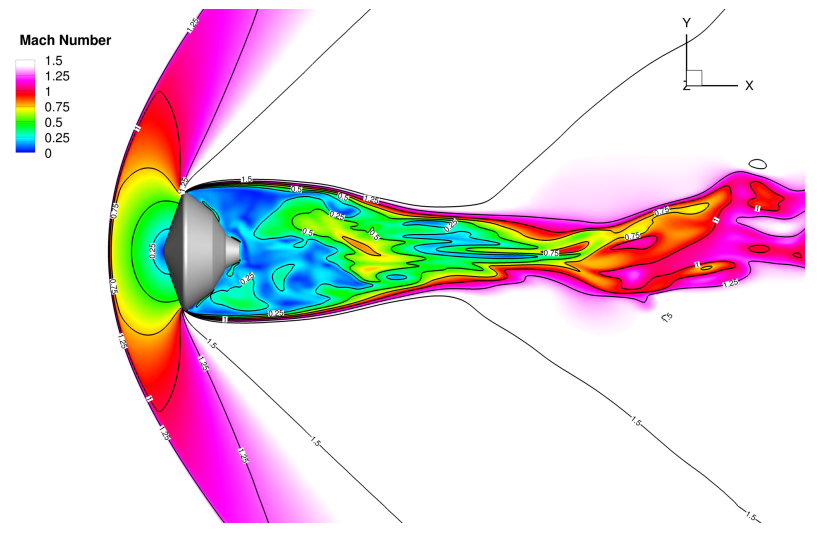

(a) Pitch plane Mach contours

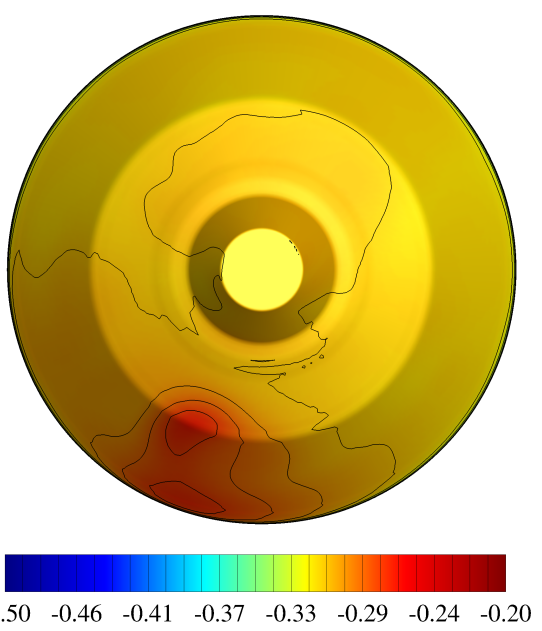

(b) Backshell pressure coefficient

Figure 31. Visualizations for Mach 1.5 and $\alpha=0^{\circ}$. 